OPEN ACCESS

Edited by:

Leonardo Julián Magnoni, Centro Interdisciplinar de Investigação Marinha e Ambiental (CIIMAR),

Portugal

Reviewed by:

Hélène Volkoff,

Memorial University of Newfoundland,

Canada

Ivan Manzini,

Justus Liebig Universität Gießen,

Germany

*Correspondence:

José L. Soengas

jsoengas@uvigo.es

Specialty section:

This article was submitted to

Aquatic Physiology,

a section of the journal

Frontiers in Physiology

Received: 01 June 2018 Accepted: 13 August 2018

Published: 28 August 2018

Citation:

Comesaña S, Velasco $C$,

Conde-Sieira M, Míguez JM, Soengas JL and Morais S (2018)

Feeding Stimulation Ability and Central Effects of Intraperitoneal

Treatment of L-Leucine, L-Valine, and L-Proline on Amino Acid Sensing Systems in Rainbow Trout: Implication

in Food Intake Control.

Front. Physiol. 9:1209.

doi: 10.3389/fphys.2018.01209

\section{Feeding Stimulation Ability and Central Effects of Intraperitoneal Treatment of L-Leucine, L-Valine, and L-Proline on Amino Acid Sensing Systems in Rainbow Trout: Implication in Food Intake Control}

\author{
Sara Comesaña ${ }^{1}$, Cristina Velasco ${ }^{1}$, Marta Conde-Sieira ${ }^{1}$, Jesús M. Míguez ${ }^{1}$, \\ José L. Soengas ${ }^{1 *}$ and Sofía Morais ${ }^{2}$
}

\begin{abstract}
'Laboratorio de Fisioloxía Animal, Departamento de Bioloxía Funcional e Ciencias da Saúde, Facultade de Bioloxía and Centro de Investigación Mariña, Universidade de Vigo, Vigo, Spain, ${ }^{2}$ Lucta S.A., Innovation Division, UAB Research Park, Bellaterra, Spain
\end{abstract}

To continue gathering knowledge on the central regulation of food intake in response to amino acids in teleost fish, using as a model rainbow trout (Oncorhynchus mykiss), we evaluated in a first experiment the feeding attractiveness of L-leucine, L-valine, and L-proline offered as an agar gel matrix. In a second experiment, we assessed the effect of intraperitoneal (IP) treatment with the same amino acids on food intake. In a third experiment, we carried out a similar IP administration of amino acids to evaluate the response of amino acid sensing mechanisms in the hypothalamus and telencephalon. Results are discussed in conjunction with an earlier study where leucine and valine were administered intracerebroventricularly (ICV). The attractiveness of amino acids does not appear to relate to their effects on food intake, at least when administrated by-passing ingestion and luminal absorption, since two attractive amino acids resulted in an anorexigenic (Leu) or no effects (Pro) on food intake while a non-attractive amino acid (Val) induced anorexigenic (IP treatment) or orexigenic (ICV treatment) responses. The effects of Leu on food intake might relate to the expression of hypothalamic neuropeptides and result from the direct activation of amino acid sensing systems. In contrast, while valine had few effects on hypothalamic amino acid sensing systems after ICV treatment, a significant amount of parameters become affected by IP treatment suggesting that the effect of Val after IP treatment is indirect. Proline had no relevant effects on amino acid sensing systems, neuropeptide expression, and food intake, which suggest that this amino acid might not have a relevant role in the homeostatic regulation of food intake through hypothalamic mechanisms. In telencephalon, the same amino acid sensing systems operating in hypothalamus appear to be present and respond to Leu and Val, but it is still unclear how they might relate to the control of food intake.

Keywords: rainbow trout, food intake, amino acid sensing, hypothalamus, telencephalon 


\section{INTRODUCTION}

The detection of changes in nutrient levels in vertebrate brain is a fundamental process involved in the homeostatic regulation of food intake (Delgado et al., 2017; Soengas et al., 2018). Homeostatic processes primarily occur in the hypothalamus, which senses metabolic and hormonal signals that inform the brain on the status of energy stores in the periphery, regulating energy intake. Accordingly, several mechanisms of nutrient sensing are present in hypothalamus to detect changes in the levels of glucose, fatty acids, and amino acids (Efeyan et al., 2015; Soengas et al., 2018). The hypothalamus contains two different neuronal populations involved in the integration of metabolic and endocrine signals related to the homeostatic control of food intake: one co-expressing appetite stimulators (orexigenic) neuropeptide Y (NPY) and Agouti-related peptide (AgRP), and another population co-expressing appetite supressors (anorexigenic) pro-opiomelanocortin (POMC) and cocaine- and amphetamine-regulated transcript (CART) (Delgado et al., 2017; Soengas et al., 2018). Thus, an increase in circulating nutrient levels results in decreased NPY/AgRP mRNA abundance, increased mRNA abundance of POMC/CART, and decreased food intake, whereas a fall in nutrient levels leads to opposite changes (Conde-Sieira and Soengas, 2017). The mechanisms linking nutrient sensors with the expression of these neuropeptides are mostly unknown in mammals (Diéguez et al., 2011) and fish (Delgado et al., 2017), although they are believed to include forkhead boxO1 (FoxO1), brain homeobox transcription factor (BSX), and phosphorylated cAMP response element binding protein (CREB).

On the other hand, the hedonic or reward-based regulation of food intake is driven by sensory perception and pleasure, resulting in consumption of highly palatable foods independently of the energy balance status, and can override the homeostatic pathway, inducing animals to overconsume food (Berthoud, 2006; Lutter and Nestler, 2009). Both homeostatic and hedonic systems are linked and activated during feeding although the degree of activation depends on the type of food and physiological status of the animal (Rossi and Stuber, 2017). In contrast to the homeostatic pathway, the mechanisms involved in the regulation of food intake by the hedonic system are scarcely known (Lutter and Nestler, 2009). The brain area in which these mechanisms are likely located in fish is the telencephalon but there are very few available studies concerning this topic (Mueller and Wullimann, 2009; O'Connell and Hofmann, 2011).

Regarding the metabolic regulation of food intake induced by changes in circulating amino acids levels in mammals, it is thought that branched-chain amino acids (BCAA) specifically signal protein availability (Efeyan et al., 2015; Heeley and Blouet, 2016). However, leucine is the unique BCAA whose increased levels are detected by mammalian central amino acid sensing mechanisms and consequently modulate NPY/AgRP and POMC/CART expression to reduce food intake (Heeley and Blouet, 2016; Soengas et al., 2018). This process occurs through not completely understood mechanisms based on (1) activation of BCAA metabolism (Morrison and Laeger, 2015), (2) activation of glutamine metabolism (Jewell and Guan, 2013), (3) activation of mechanistic target of rapamycin (mTOR) (Cavanaugh et al., 2015; Hu et al., 2016) and/or inhibition of AMP-activated protein kinase (AMPK) signaling (Fromentin et al., 2012), and (4) umami taste receptor signaling (Wauson et al., 2012). Taste receptors of the type 1 (T1R) family, beyond their sensory function in oral tissues, are also expressed in brain, where they play an important role as nutrient sensors (Herrera Moro Chao et al., 2016; Lee and Owyang, 2017). Furthermore, there is another mechanism which is activated in cases of deficiency of essential amino acids and elicits an increase in food intake mediated by general control non-derepressible 2 (GCN2) kinase which subsequently phosphorylates eukaryotic initiation factor $2 \alpha$ (eIF2 $\alpha$ ), leading to reduced global protein synthesis and increased expression of specific genes. One of these genes is system A amino acid transporter 2 (SNAT2), which has been considered to detect changes in BCAA alone, i.e., without previous changes in GCN2 (Hundal and Taylor, 2009). Another gene is sestrin 2 (SESN2), which has been postulated to inhibit mTOR in the absence of leucine (Wolfson and Sabatini, 2017).

In a previous study (Comesaña et al., 2018) on rainbow trout (Oncorhynchus mykiss) as a model teleost fish, we demonstrated that intracerebroventricular (ICV) treatment with leucine decreased food intake as expected whereas valine, contrary to mammals, had a clear orexigenic effect. We related the effects on food intake to the presence of amino acid sensing systems (dependent on metabolism of BCAA, metabolism of glutamine, mTOR, T1R receptors, and GCN2) sensitive to leucine in hypothalamus and telencephalon. As for valine, responses of amino acid sensing systems were partially observed in telencephalon but not in hypothalamus. This earlier study led us to hypothesize that valine might be attractive to trout and that stimulation of food intake might have occurred through reward and hedonic mechanisms operating in telencephalon.

Therefore, the first objective of this follow-up study was to confirm the hypothesized hedonic value of valine by performing a feeding attractiveness experiment with amino acids offered as an agar gel matrix, in order to relate the central regulation of food intake by amino acids to their possible feeding stimulation effects. In a second experiment, we assessed the effect of intraperitoneal (IP) treatment with leucine and valine on food intake. These amino acids were initially selected considering that, in mammals, leucine is the unique activator of amino acid sensing systems in brain whereas valine acts as a negative control (Heeley and Blouet, 2016). However, the previous realization of important differences between trout and mammals (Comesaña et al., 2018) led us to consider extending the investigation to a non-BCAA in this study. Proline was was selected for several reasons: it typically has a high palatability in carnivore fish species (Li et al., 2009; Morais, 2017), including rainbow trout (Jones, 1989), it has very diverse and important roles in cell metabolism and physiology, and is considered a conditionally essential amino acid in fish given that rates of endogenous synthesis are inadequate during early life stages, and possibly also in adults (Li et al., 2009; Wu et al., 2011). Furthermore, the requirement of proline for whole-body protein synthesis is the highest of all amino acids, on a per-gram basis (Wu et al., 2011). Finally, in order to establish whether the action of dietary leucine and valine on central amino 
acid sensors is possibly direct, we carried out a third experiment with a similar IP administration of amino acids to evaluate the response of amino acid sensing mechanisms in the hypothalamus and telencephalon, and compare it with the previous results after ICV administration (Comesaña et al., 2018). In particular, we assessed parameters related to putative amino acid sensing mechanisms based on: (1) metabolism of BCAA, evaluated through branched-chain amino acid aminotransferase (BCAT) activity, and mRNA abundance of branched-chain $\alpha$-keto acid dehydrogenase E2 subunit (BCKDE2) and branched chain ketoacid dehydrogenase kinase (BCKDK), (2) metabolism of glutamine, evaluated by glutamine synthase (GLS) and glutamate dehydrogenase $(\mathrm{GDH})$ activities, and mRNA abundance of GLS1 and GLS2, (3) mTOR/AMPK $\alpha$, evaluated by the abundance of their mRNA as well as by their phosphorylation status, (4) $\mathrm{T} 1 \mathrm{R}$ receptors, including the umami and sweet taste receptors (which in fish also bind amino acid ligants; Oike et al., 2007), through mRNA abundance of subunits T1R1, T1R2, and T1R3, and (5) GCN2, evaluated by mRNA abundance of eIF2 $\alpha$, SNAT2, and SESN2. Additionally, we also evaluated changes in mRNA abundance of neuropeptides involved in food intake regulation (NPY, AgRP, POMC-A1, and CART), as well as levels and phosphorylation status of proteins (CREB and FoxO1) putatively involved in linking changes in amino acid sensing systems with the expression of neuropeptides. In sum, the experiments reported here will enable to continue gathering knowledge on the central regulation of food intake in response to amino acids in a teleost fish.

\section{MATERIALS AND METHODS}

\section{Fish}

Rainbow trout were obtained from a local fish farm (A Estrada, Spain) and maintained under laboratory conditions in the Universidade de Vigo for 1 month in $100 \mathrm{~L}$ tanks, with 12L:12D photoperiod (lights on at 08:00 h, lights off at 20:00 h), in dechlorinated tap water at $15^{\circ} \mathrm{C}$. Fish were fed once daily $(09.00 \mathrm{~h})$ to satiety with commercial dry fish pellets (Dibaq-Diproteg SA, Spain) containing 48\% crude protein, 14\% carbohydrates, $25 \%$ crude fat, $11.5 \%$ ash, and $20.2 \mathrm{MJ} / \mathrm{kg}$ of feed. The experiments described comply with the Guidelines of the European Union Council (2010/63/UE), and of the Spanish Government (RD 55/2013) for the use of animals in research, and were approved by the Ethics Committee of the Universidade de Vigo.

\section{Experimental Design Feeding Stimulation}

Following 1-month acclimation period, fish of $87.6 \pm 8.3 \mathrm{~g}$ size were randomly assigned to eight $100 \mathrm{~L}$ experimental tanks at a density of four fish per tank. In this first experiment, fish were offered pellets of an agar gel matrix containing water alone (control) or with L-leucine, L-valine, or L-proline, as described by Papatryphon and Soares (2000). The test gels were prepared solubilizing agar-agar by heating at a concentration of $2 \%$ in $40 \mathrm{~mL}$ of deionized water. Before reaching gelatinization, L-leucine, L-valine, or L-proline were added at a final concentration of $0.1 \mathrm{M}$, and a red dye (4R Ponceau, \#199737, Sigma, St. Louis, MO, United States) was added to all gels at a final concentration of $5 \mu \mathrm{M}$, to enable visual detection of the pellets. In the control group, the pellets contained only the dye. The gel was poured into Petri dishes and allowed to completely gelatinize. The pellets were cut out to similar size pieces of $3 \mathrm{~mm}$ diameter and $3 \mathrm{~mm}$ length using a stainless steel tube immediately before feeding. Fish were fed twice daily with the agar pellets in the morning offering fish the same amount of pellets (in excess) in all treatments, when food intake was recorded, and with commercial dry fish pellets (see above) in the afternoon. Agar pellets offered were counted and uneaten pellets were withdrawn and counted to know the number of pellets consumed by all fish in each tank. The test diets were rotated among the tanks every day during 8 days, so that each tank received each tested diet twice. Two replicates of each of the four groups were tested each day, resulting in $n=16$ tests per treatment. Taste attractiveness of each amino acid was expressed as \% of pellets ingested/pellets offered.

\section{IP Injection}

A second type of experiments was performed using new fish acclimated for at least 1 month and randomly assigned to four $100 \mathrm{~L}$ experimental tanks. Fish were fasted for $24 \mathrm{~h}$ before treatment to ensure that basal levels of hormones involved in metabolic control were achieved. On the day of the experiment, fish were lightly anesthetized with 2-phenoxyethanol (Sigma, $0.02 \% \mathrm{v} / \mathrm{v})$ and weighed. Fish received $0.5 \mathrm{~mL} \cdot 100 \mathrm{~g}^{-1}$ IP injection of saline solution $(0.6 \% \mathrm{NaCl})$ alone (control) or containing $40 \mu \mathrm{mol} \cdot \mathrm{mL}^{-1} \mathrm{~L}$-leucine, $\mathrm{L}$-valine, or L-proline. Dose was calculated from the amount of leucine ingested per day by a trout fed a standard commercial diet (Wacyk et al., 2012).

In a first set of experiments, fish of $89.2 \pm 3.3 \mathrm{~g}$ size were used for the assessment of food intake. This was registered in the whole tank for 3 days before treatment (to evaluate basal level of food intake) and then 6,24 , and $48 \mathrm{~h}$ after IP treatment with saline solution alone (control, $n=10$ fish) or containing L-leucine ( $n=10$ fish), L-valine ( $n=10$ fish), or L-proline ( $n=10$ fish). After feeding, uneaten food and feed waste remaining at the bottom of the conical tanks were withdrawn, dried, and weighed, and this value was used to calculate the amount of food consumed by all fish in each tank, as the difference from the feed offered (de Pedro et al., 1998; Polakof et al., 2008a,b). The experiment was repeated three times and results shown are the mean \pm SEM of the three experiments $(N=3)$ each with $n=10$ fish per treatment in each tank.

In a second set of experiments, fish of $71.8 \pm 1.3 \mathrm{~g}$ size were IP injected with saline solution alone (control, $n=22$ fish) or containing L-leucine $(n=22$ fish), L-valine $(n=22$ fish), or L-proline ( $n=22$ fish), as described above. After 6 h, fish were lightly anesthetized with 2-phenoxyethanol (Sigma, $0.02 \% \mathrm{v} / \mathrm{v}$ ). Blood was collected by caudal puncture with ammonium-heparinized syringes, and plasma samples were obtained after blood centrifugation, deproteinized immediately (using 0.6 M perchloric acid), and neutralized (using $1 \mathrm{M}$ potassium bicarbonate) before freezing on dry ice and storing 
at $-80^{\circ} \mathrm{C}$ until further assay. Fish were then sacrificed by decapitation, and hypothalamus and telencephalon were dissected, snap-frozen, and stored at $-80^{\circ} \mathrm{C}$. Ten fish per group were used to measure enzyme activities and metabolite levels, six fish per group were used for the assessment of mRNA levels by qRT-PCR, whereas the remaining six fish per group were used to assess changes in the phosphorylation status of proteins through western blot.

\section{Assessment of Metabolite Levels and Enzyme Activities}

Levels of glucose and lactate in plasma were determined enzymatically using commercial kits (Spinreact, Barcelona, Spain). Total $\alpha$-amino acids were assessed colorimetrically using the nynhydrin method (Moore, 1968) with alanine as standard.

Samples used to assess tissue metabolite levels were quickly homogenized by ultrasonic disruption in 7.5 vols of ice-cooled $0.6 \mathrm{M}$ perchloric acid, and neutralized with $1 \mathrm{M}$ potassium bicarbonate. The homogenate was centrifuged $(10,000 \mathrm{~g})$ and the supernatant used to assay tissue metabolites. Tissue total $\alpha$-amino acids levels were determined colorimetrically as described above for plasma samples.

Samples for enzyme activities were homogenized by ultrasonic disruption with 9 vols of ice-cold buffer consisting of $50 \mathrm{mM}$ Tris ( $\mathrm{pH}$ 7.6), $5 \mathrm{mM}$ EDTA, $2 \mathrm{mM}$ 1,4-dithiothreitol, and a protease inhibitor cocktail (Sigma). The homogenate was centrifuged and the supernatant used immediately for enzyme assays. Enzyme activities were determined using a microplate reader INFINITE 200 Pro (Tecan, Männedorf, Switzerland). Reaction rates of enzymes were determined by the decrease in absorbance of $\mathrm{NADH}$ at $340 \mathrm{~nm}$ or, in the case of GLS activity, of Fe-G-hydroxy glutamyl complex at $500 \mathrm{~nm}$ in acid medium, for which $\mathrm{FeCl}_{3} \quad 6.7 \%$ in $\mathrm{HCl} 1 \mathrm{~N}$ was added after incubation. The reactions were started by the addition of supernatant $(10-15 \mu \mathrm{L})$ at a pre-established protein concentration, omitting the substrate in control wells (final volume 180-275 $\mu \mathrm{L}$ ), and allowing the reactions to proceed at $37^{\circ} \mathrm{C}$ for pre-established times (3-20 $\mathrm{min}$ ). Enzyme activities were normalized to protein levels (mg). Protein was assayed in triplicate in homogenates using a microplate reader, according to the bicinchoninic acid method with bovine serum albumin (Sigma) as standard. Enzyme activities were assessed at maximum rates after preliminary tests to determine optimal substrate concentrations. BCAT (EC 2.6.1.42), glutamine synthetase (GLS, EC 6.3.1.2), and GDH (EC 1.4.1.4) activities were determined as previously described (Comesaña et al., 2018).

\section{Analysis of mRNA Abundance by Real-Time Quantitative PCR}

Total RNA of hypothalamus and telencephalon samples was extracted using Trizol reagent (Life Technologies, Grand Island, NY, United States) and subsequently treated with RQ1-DNAse (Promega, Madison, WI, United States); $2 \mu \mathrm{g}$ total RNA were reverse transcribed using Superscript II reverse transcriptase (Promega) and random hexamers (Promega) in a reaction volume of $20 \mu \mathrm{L}$. Gene expression levels were determined by real-time quantitative PCR (RT-qPCR) using the iCycler iQ (BIO-RAD, Hercules, CA, United States). Analyses were performed on $1 \mu \mathrm{L}$ cDNA (previously diluted 1:1) using MAXIMA SYBR Green qPCR Mastermix (Life Technologies), in a total PCR reaction volume of $15 \mu \mathrm{L}$, containing $50-500 \mathrm{nM}$ of each primer. Most transcripts were measured using previously described primers (Comesaña et al., 2018), with the exception of T1R1 and SESN2. For these transcripts, new primers were designed using Primer3 software ${ }^{1}$ from sequences available in GenBank (T1R1, XM_021614421.1; SESN2, XM_021572426.1). A fragment of each sequence containing the amplicon was amplified by conventional PCR and run on a 1.2\% agarose gel. The corresponding bands were cut from the gel, purified with the QIAquick Gel Extraction Kit (Qiagen, Hilden, Germany) and sequenced in an Applied Biosystems 3130 (Foster City, CA, United States) in Servicio de Determinación Estructural, Proteómica y Genómica (CACTI-Universidade de Vigo). The obtained sequences satisfactorily matched the reference GenBank sequences. Forward and reverse primers used for each gene expression assay are shown in Supplementary Table S1. Thermal cycling was initiated with incubation at $95^{\circ} \mathrm{C}$ for $90 \mathrm{~s}$ using hot-start iTaq DNA polymerase activation followed by 40 cycles, each one consisting of heating at $95^{\circ} \mathrm{C}$ for $20 \mathrm{~s}$, and specific annealing and extension temperatures (Supplementary Table S1) for $20 \mathrm{~s}$. Following the final PCR cycle, melting curves were systematically performed and monitored $\left(55^{\circ} \mathrm{C}\right.$ temperature gradient at $0.5^{\circ} \mathrm{C} / \mathrm{s}$ from 55 to $94^{\circ} \mathrm{C}$ ) to ensure that only one fragment was amplified. Samples without reverse transcriptase and samples without RNA were run in each qPCR assay as negative controls. Relative expression of the target transcripts was calculated using $\beta$-actin and elongation factor $1 \alpha(\mathrm{EF} 1 \alpha)$ as reference genes, which were stably expressed in this experiment, following the Pfaffl (2001) method.

\section{Western Blot Analysis}

Frozen samples were homogenized in $1 \mathrm{~mL}$ of buffer containing $150 \mathrm{mM} \mathrm{NaCl}, 10 \mathrm{mM}$ Tris- $\mathrm{HCl}, 1 \mathrm{mM}$ EGTA, $1 \mathrm{mM}$ EDTA ( $\mathrm{pH}$ 7.4), $100 \mathrm{mM}$ sodium fluoride, $4 \mathrm{mM}$ sodium pyrophosphate, $2 \mathrm{mM}$ sodium orthovanadate, $1 \%$ Triton $\mathrm{X}$ $100,0.5 \%$ NP40-IGEPAL, and $1.02 \mathrm{mg} \cdot \mathrm{mL}^{-1}$ protease inhibitor cocktail (Sigma). Tubes were kept on ice during the whole process to prevent protein denaturation. Homogenates were centrifuged at $1,000 \mathrm{~g}$ for $15 \mathrm{~min}$ at $4^{\circ} \mathrm{C}$, and supernatants were again centrifuged at $20,000 \mathrm{~g}$ for $30 \mathrm{~min}$. The resulting supernatants were recovered and stored at $-80^{\circ} \mathrm{C}$. The concentration of protein in each sample was determined using the Bradford assay with bovine serum albumin as standard. Protein lysates $(20 \mu \mathrm{g})$ were used for western blotting using appropriate antibodies from (1) Cell Signaling Technology (Leiden, Netherlands): anti-phospho AMPK $\alpha$ (Thr172) ref. \#2531, anti-AMPK $\alpha$ ref. \#2532, anti-CREB (48H2) ref. \#9197, anti-phospho-CREB (Ser133) ref. \#9198, anti-phospho-FoxO1 (Thr24) \#ref. 9464, anti-FoxO1 (L27) ref. \#9454, anti-phosphomTOR (Ser2448) ref. \#5536, and anti- $\beta$-tubulin ref. \#2146, or (2) Sigma: anti m-TOR ref. \#T2949. All these antibodies

\footnotetext{
${ }^{1}$ http://bioinfo.ut.ee/primer3-0.4.0/primer3/
} 
cross-react successfully with the proteins of interest in rainbow trout (Skiba-Cassy et al., 2009; Sánchez-Gurmaches et al., 2010; Kamalam et al., 2012; Librán-Pérez et al., 2015; Velasco et al., 2016). After washing, membranes were incubated with an IgGHRP secondary antibody ref. \#2015718 (Abcam, Cambridge, United Kingdom) and bands were quantified by Image Lab software version 5.2.1 (BIO-RAD) in a Chemidoc Touch Imaging system (BIO-RAD).

\section{Statistics}

Comparisons among groups were carried out with one-way ANOVA followed by a Student-Newman-Keuls test using the statistical package SigmaStat. Differences were considered statistically significant at $P<0.05$.

\section{RESULTS}

When fish were offered agar pellets containing $0.1 \mathrm{M}$ of either L-leucine, L-valine, and L-proline, a significantly higher ingestion was obtained with L-leucine and L-proline, which was particularly remarkable for L-leucine, compared with the control group, while L-valine was ingested at a similar level as the control (Figure 1).

In the second experiment, food intake decreased significantly $24 \mathrm{~h}$ after IP administration with leucine and valine, and remained significantly lower $48 \mathrm{~h}$ after treatment with valine, compared to the control and proline treatment. IP injection with proline, on the other hand, did not affect subsequent food intake (Figure 2).

Total $\alpha$-amino acids in plasma (Figure 3A) were not significantly different compared with the control, but the proline group showed decreased values compared with the valine-treated group. Both leucine and proline treatment led to a significant decrease in plasma glucose (Figure 3B), compared to the control

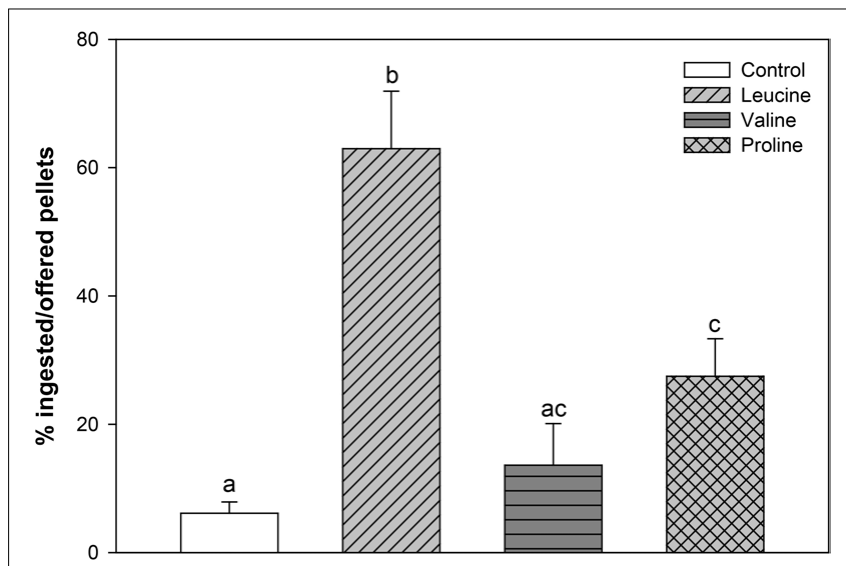

FIGURE 1 | Percentage of ingested pellets, in relation to offered pellets, of rainbow trout fed agar pellets containing water alone (control) or $0.1 \mathrm{M}$ L-leucine, L-valine, or L-proline. The results are shown as mean + SEM of 16 different experiments in which four fish were used per group in each tank. Different letters indicate significant differences $(P<0.05)$ between different groups. $P$-values are: $C$ vs $L<0.001, C$ vs $V=0.511, C$ vs $P=0.012, L$ vs $V<0.001, L$ vs $P=0.005, V$ vs $P=0.142$ and valine treatment, and no changes in lactate level compared to the control. Valine had an opposite effect, significantly reducing lactate level in plasma compared to the control, leucine, and proline (only numerically in this case) group (Figure 3C).

The levels of $\alpha$-amino acids increased in hypothalamus (Figure 4A) with proline treatment compared with the control, while in telencephalon (Figure $4 \mathbf{B}$ ), both valine and proline treatments increased the levels of $\alpha$-amino acids compared with the control group.

The mRNA abundance of neuropeptides is shown in Figure 5. In hypothalamus, leucine treatment induced an increase in POMC-A1 expression (Figure 5C) compared with the control group, $6 \mathrm{~h}$ after IP administration. In the same tissue, valine treatment decreased mRNA levels of NPY (Figure 5A) and increased POMC-A1 expression (Figure 5C). Treatment with proline did not affect the expression of neuropeptides in hypothalamus. In telencephalon, the valine group showed significantly increased levels of NPY compared with the control and leucine groups (Figure 5E), whereas leucine and proline IP administration did not significantly affect mRNA abundance of neuropeptides in this tissue.

The parameters assessed related to BCAA metabolism are shown in Figure 6. The activity of BCAT in hypothalamus was lower in the group treated with proline than in the leucine treatment (Figure 6A). Leucine treatment induced a significant increase in hypothalamic mRNA abundance of BCKDE2 in relation to the control group (Figure 6B). In telencephalon, relative to the control, leucine treatment increased the expression of BCKDE2 (Figure 6E), whereas valine treatment increased mRNA abundance of both BCKDE2 (Figure 6E) and BCKDK (Figure 6F).

Figure 7 depicts parameters related to glutamate and glutamine metabolism. In the hypothalamus, GLS activity decreased in the valine-treated group compared with the control group (Figure 7B). In telencephalon, GDH activity was significantly higher $6 \mathrm{~h}$ after IP treatment with proline compared with the other groups (Figure 7E), while IP treatment with leucine and valine significantly increased mRNA abundance of GLS2 (Figure 7H).

The expression and phosphorylation status of mTOR and $\mathrm{AMPK} \alpha$ are displayed in Figure 8. In hypothalamus, leucine treatment was responsible for a significant increase in mTOR phosphorylation status (Figure 8B) and AMPK $\alpha 1$ mRNA abundance (Figure 8C) compared with the control. Valine treatment, on the other hand, increased both mRNA abundance (Figure 8A) and phosphorylation status (Figure 8B) of mTOR, while proline treatment increased mRNA abundance (Figure 8C) and phosphorylation status (Figure 8D) of AMPK $\alpha$. In telencephalon, the only significant effect was observed in mTOR expression, which was higher in valine IP-treated fish than in the other groups (Figure 8E).

The parameters related to mechanisms mediated by GCN2 are shown in Figure 9. In hypothalamus, SNAT2 mRNA abundance was significantly increased in the leucine-treated group compared with the remaining treatments (Figure 9B). In telencephalon, eIF2 $\alpha$ expression (Figure 9D) was significantly higher in leucine, valine, and proline-treated groups than in the control. 


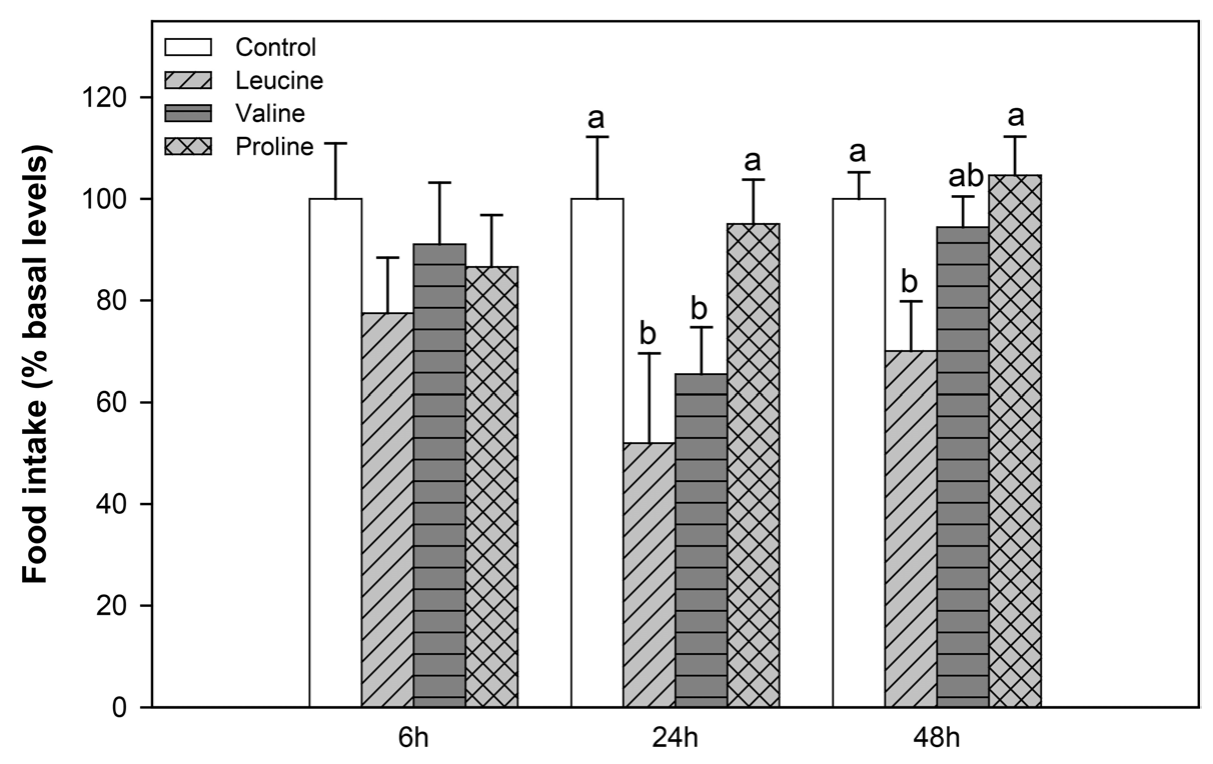

FIGURE 2 | Food intake in rainbow trout 6, 24, and $48 \mathrm{~h}$ after intraperitoneal administration of $0.5 \mathrm{~mL} \cdot 100 \mathrm{~g}^{-1}$ body mass of saline solution alone (control) or containing $40 \mu \mathrm{mol} \cdot \mathrm{mL}^{-1}$ of L-leucine, $40 \mu \mathrm{mol} \cdot \mathrm{mL}^{-1}$ of L-valine, or $40 \mu \mathrm{mol} \cdot \mathrm{mL}^{-1}$ of L-proline. Food intake is displayed as mean $+\mathrm{SEM}$ of the percentage of food ingested with respect to baseline levels (calculated as the average of food intake in the 3 days previous to the experiment), from three different experiments in which 10 fish were used per group in each tank. Different letters indicate significant differences $(P<0.05)$ between different groups. $P$-values are: at $6 \mathrm{~h}$, $=0.389$; at $24 \mathrm{~h}$, $C$ vs $L=0.017, C$ vs $V=0.030, C$ vs $P=0.838, L$ vs $V=0.412, L$ vs $P=0.015, V$ vs $P=0.029 ;$ and at $48 \mathrm{~h}, C$ vs $L=0.037, C$ vs $V=0.817, C$ vs $P=0.869, L$ vs $V=0.237, L$ vs $P=0.039, V$ vs $P=0.639$.

Expression of T1R taste receptors was also measured, and is shown in Figure 10. Only the expression of T1R3 was significantly affected, in both brain regions: in hypothalamus, mRNA abundance of T1R3 was increased by leucine and valine treatment (Figure 10C) compared with the control group, while in telencephalon, only valine treatment enhanced T1R3 expression, above the remaining groups (Figure 10F).

Finally, Figure 11 displays the phosphorylation status of CREB and FoxO1. Significantly differences were only found in hypothalamus, where the ratio of phoshorylated and unphosphorylated forms of CREB decreased in the groups treated with leucine and proline (and tendentially in the valine group) compared with the control (Figure 11B).

\section{DISCUSSION}

\section{Differential Amino Acid Attractiveness Inducing Differences in Feed Intake}

Amino acids are important attractants and feeding effectors in all teleost fishes, being detected both by the sense of smell and taste. Olfaction tends to be a more evolutionarily conserved response, involved in other basic functions besides feeding (e.g., kinnonkin recognition, prey-predator interactions, and territory or homing recognition), while the gustatory system is considered the main determinant of the feeding behavior, providing the final evaluation of the sensorial and nutritional properties of the food, and determining its final consumption (Goh and Tamura, 1980; Hara, 1994). For this reason, the taste spectra of amino acids eliciting stimulatory or deterrent feeding responses is highly species-specific (Hara, 1994; Morais, 2017). Salmonids, including rainbow trout, belong to a group of teleosts which show a narrow range of taste response, responding only to a few amino acids (Marui et al., 1983; Hara, 1994; Kohbara and Caprio, 2001).

Previously (Comesaña et al., 2018), we described the presence of central amino acid sensing mechanisms in rainbow trout brain involved in the regulation of food intake, sharing some similarities with the mammalian system. However, the ICV treatment with valine had a clear and surprising orexigenic effect that was not related to the activation of amino acid sensing in hypothalamus but might have involved the telencephalon, which has been suggested as a possible center for hedonic or reward-based regulation of food intake in fish (Mueller and Wullimann, 2009; O'Connell and Hofmann, 2011). Contrary to leucine, valine has not been previously reported as stimulating feed intake in rainbow trout (Jones, 1989), but studies evaluating its attractiveness are scarce and variation of gustatory specificity of different strains of rainbow trout to amino acid stimuli has been reported (Hara et al., 1999). Therefore, we decided to evaluate the feeding response to leucine and valine by performing a feeding trial in the present study, which should link to their hedonic value. We have also extended this work to include proline, as it has been reported as the most potent amino acid eliciting a gustatory response in both electrophysiological (Marui et al., 1983; Kohbara and Caprio, 2001) and behavioral (Jones, 1989) studies with rainbow trout.

Our results indicate that $0.1 \mathrm{M}$ of leucine, followed by proline, stimulated the consumption of agar pellets, whereas valine did not affect feeding. These results are consistent with a previous feeding behavioral study, where both L-proline and L-leucine 

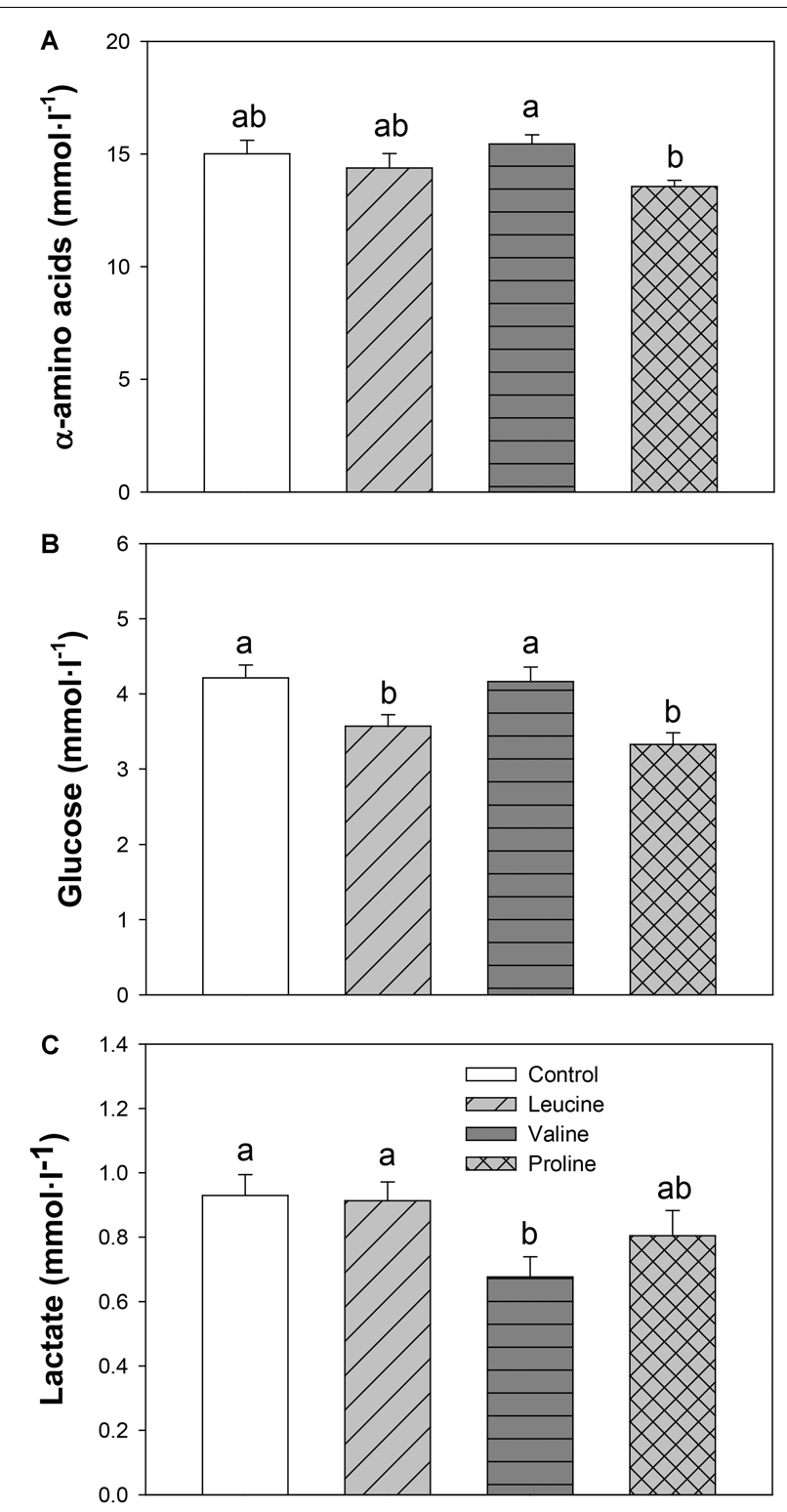

FIGURE 3 | Plasma levels of $\alpha$-amino acids (A), glucose (B), and lactate (C) in rainbow trout $6 \mathrm{~h}$ after intraperitoneal administration of $0.5 \mathrm{~mL} \cdot 100 \mathrm{~g}^{-1}$ body mass of saline solution alone (control) or containing $40 \mu \mathrm{mol} \cdot \mathrm{mL}^{-1}$ of L-leucine, $40 \mu \mathrm{mol} \cdot \mathrm{mL}^{-1}$ of L-valine, or $40 \mu \mathrm{mol} \cdot \mathrm{mL}^{-1}$ of L-proline. Each value is the mean + SEM of $n=22$ fish per treatment. Different letters indicate significant differences $(P<0.05)$ between different groups. $P$-values are: for $\alpha$-amino acids (A), $C$ vs $L=0.364, C$ vs $V=0.524, C$ vs $P=0.096, L$ vs $V=0.267, L$ vs $P=0.240, V$ vs $P=0.035$; for glucose (B), $C$ vs $L=0.019, C$ vs $V=0.832, C$ vs $P=0.002, L$ vs $V=0.013, L$ vs $P=0.305, V$ vs $P=0.002$; and for lactate (C), $C$ vs $L=0.856, C$ vs $V=0.030, C$ vs $P=0.364, L$ vs $V=0.027, L$ vs $P=0.240, V$ vs $P=0.164$

were highly effective gustatory compounds, while there was no response to valine (Jones, 1989). The higher response to leucine than to proline was somewhat surprising but this could be related to differences in sensitivity (i.e., threshold of activation of taste nerves) to these amino acids. For instance, proline was the most effective compound at $10^{-4} \mathrm{M}$ but was not found
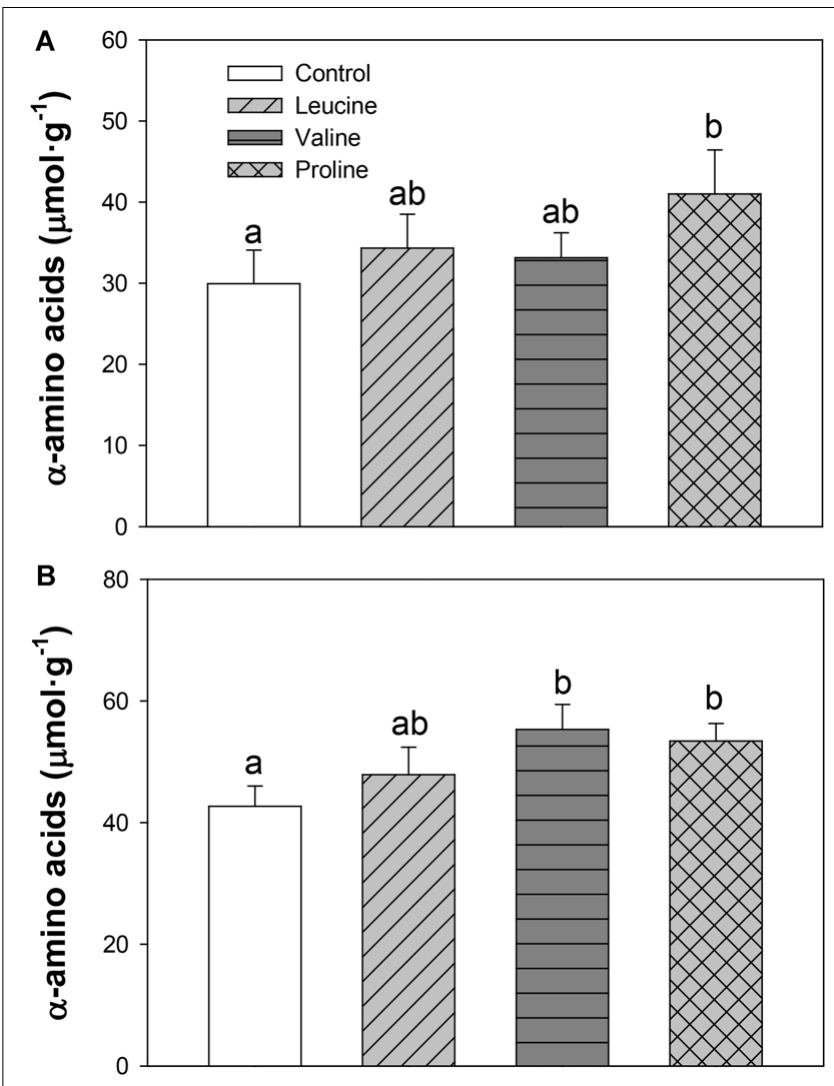

FIGURE 4 | Levels of $\alpha$-amino acids in hypothalamus (A) and telencephalon (B) of rainbow trout $6 \mathrm{~h}$ after intraperitoneal administration of $0.5 \mathrm{~mL} \cdot 100 \mathrm{~g}^{-1}$ body mass of saline solution alone (control) or containing $40 \mu \mathrm{mol} \cdot \mathrm{mL}^{-1}$ of L-leucine, $40 \mu \mathrm{mol} \cdot \mathrm{mL}^{-1}$ of L-valine, or $40 \mu \mathrm{mol} \cdot \mathrm{mL}^{-1}$ of L-proline. Each value is the mean + SEM of $n=10$ fish per treatment. Different letters indicate significant differences $(P<0.05)$ between different groups. $P$-values are: for hypothalamus (A), $C$ vs $L=0.448, C$ vs $V=0.514, C$ vs $P=0.037, L$ vs $V=0.809, L$ vs $P=0.149, V$ vs $P=0.287$; and for telencephalon (B), $C$ vs $L=0.338, C$ vs $V=0.024, C$ vs $P=0.020, L$ vs $V=0.212, L$ vs $P=0.286, V$ vs $P=0.698$.

active at $10^{-5} \mathrm{M}$ (Jones, 1989). Therefore, our previous results with valine (Comesaña et al., 2018) cannot be justified by the activation of a putative reward system in the telencephalon and remain unexplained at present. Clearly, many more studies are necessary on the different brain areas and mechanisms possibly regulating food intake in fish in response to amino acids.

\section{Effects of Amino Acids on Food Intake and Neuropeptide mRNA Abundance}

Important differences were observed in the response of several of the parameters assessed in this study (discussed in this and following sections), not only in relation to the control but also between the different amino acid treatments, which validates that the amino acids administered via IP reached the brain in sufficient concentration.

The IP treatment with leucine resulted in a prolonged decrease in food intake ( 24 and $48 \mathrm{~h}$ post-treatment), while valine reduced 


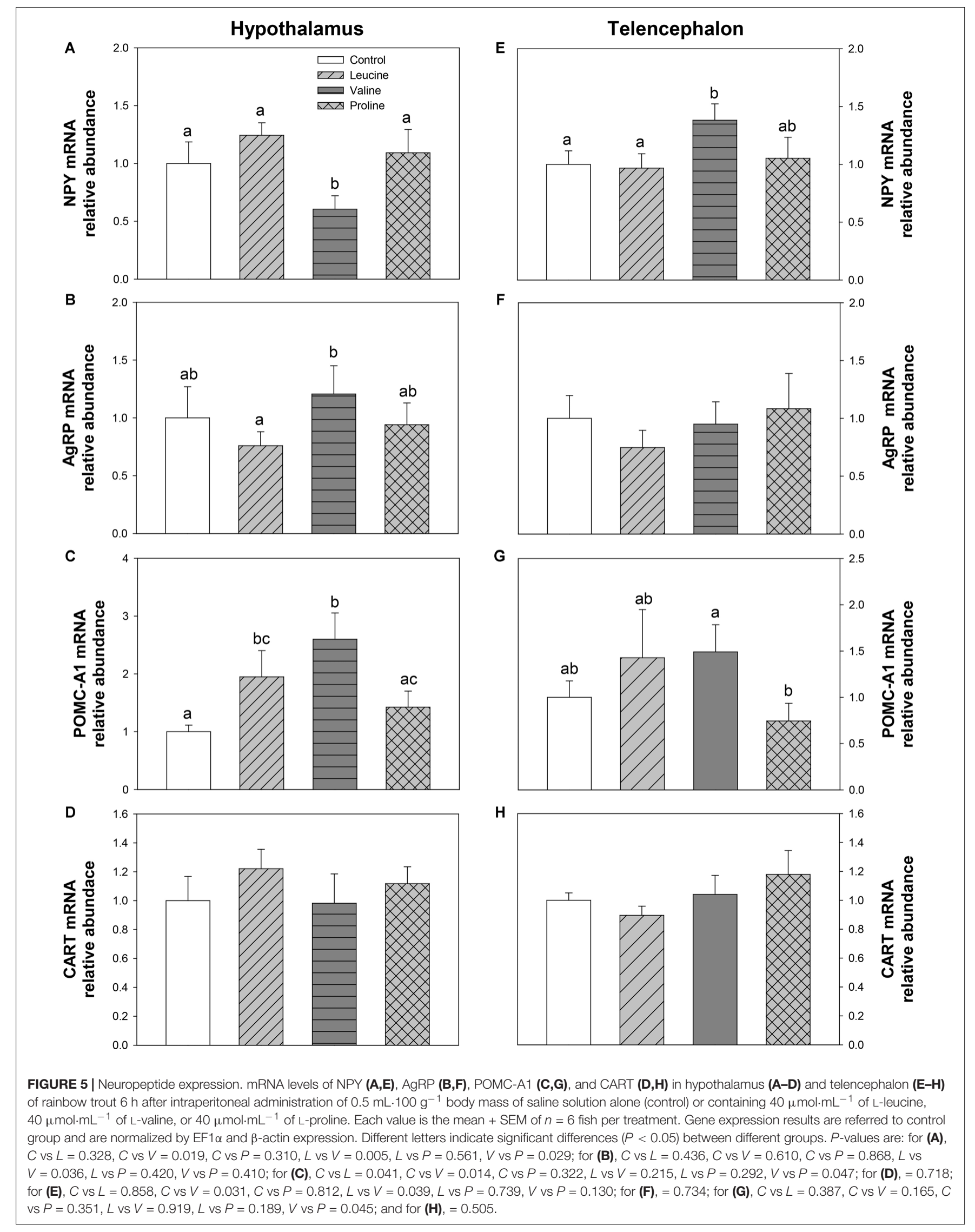




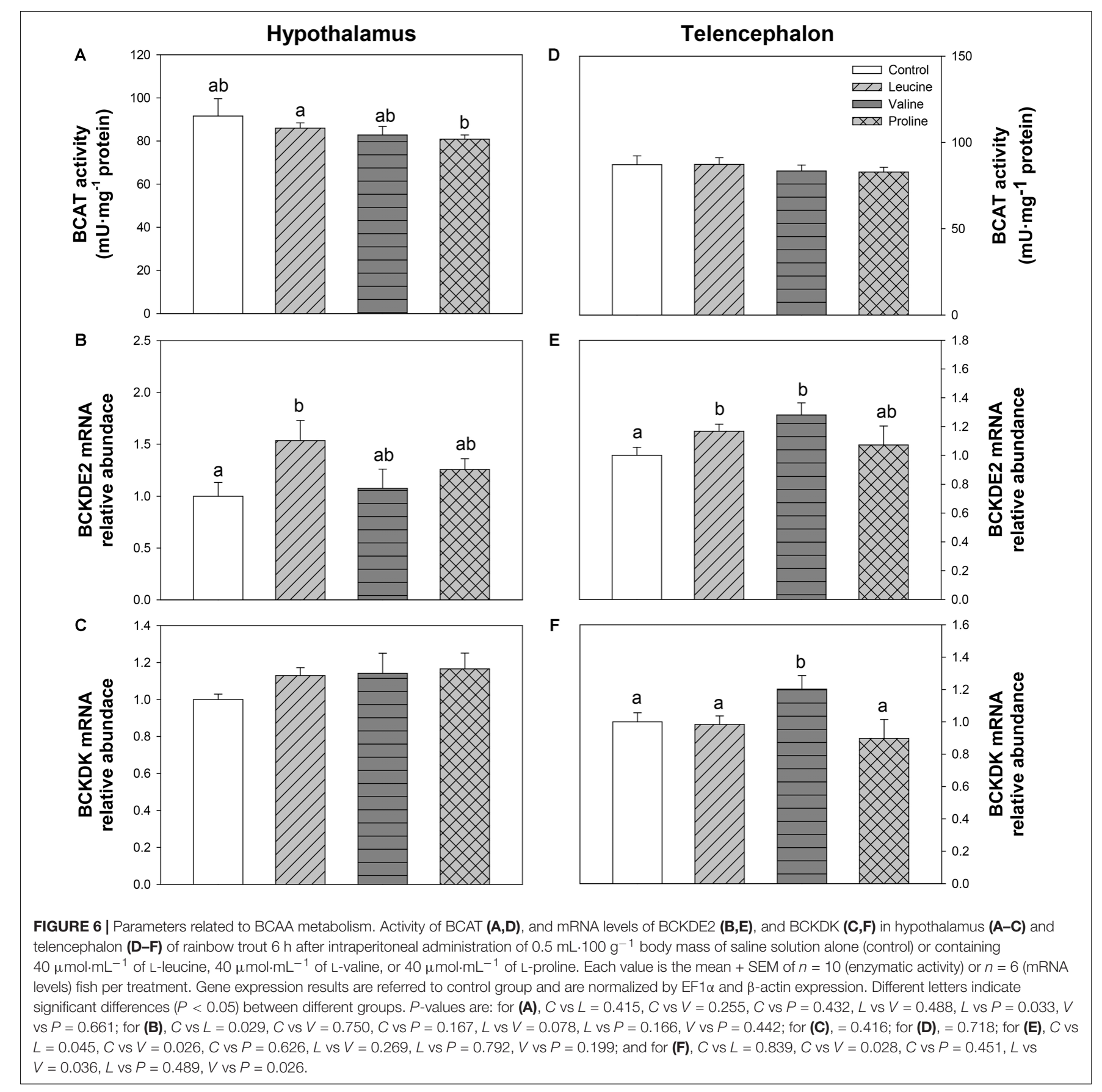

food intake $24 \mathrm{~h}$ after treatment. Administration of proline via IP, on the other hand, did not affect food intake at the times assessed. No other studies are available in fish regarding the effects of IP treatment with any amino acid on food intake, but if we compare these results with those after ICV treatment (Comesaña et al., 2018), we observe that leucine produces similar inhibitory effects, whereas valine has contrary effects. It would therefore appear that leucine functions as a signal of nutrient or energy availability to reduce food intake, whereas valine might have different roles. It is important to highlight that valine has no effects on food intake when administered ICV in mammals (Cota et al., 2006; Blouet and Schwartz, 2012) and that physiological changes in plasma leucine concentration do not lead to a decrease in food intake in mammals (Jordi et al., 2013), even if brain leucine levels are well established as an anorectic signal (Heeley and Blouet, 2016). Therefore, even if amino acid sensing systems are operational in fish and coupled to neuropeptide expression, as in mammals, their regulation of food intake in response to circulating amino acids is clearly different.

Interestingly, food intake after IP treatment with amino acids was not related to their attractiveness, since both leucine, which was highly efficient in inducing feeding, and valine, which did 


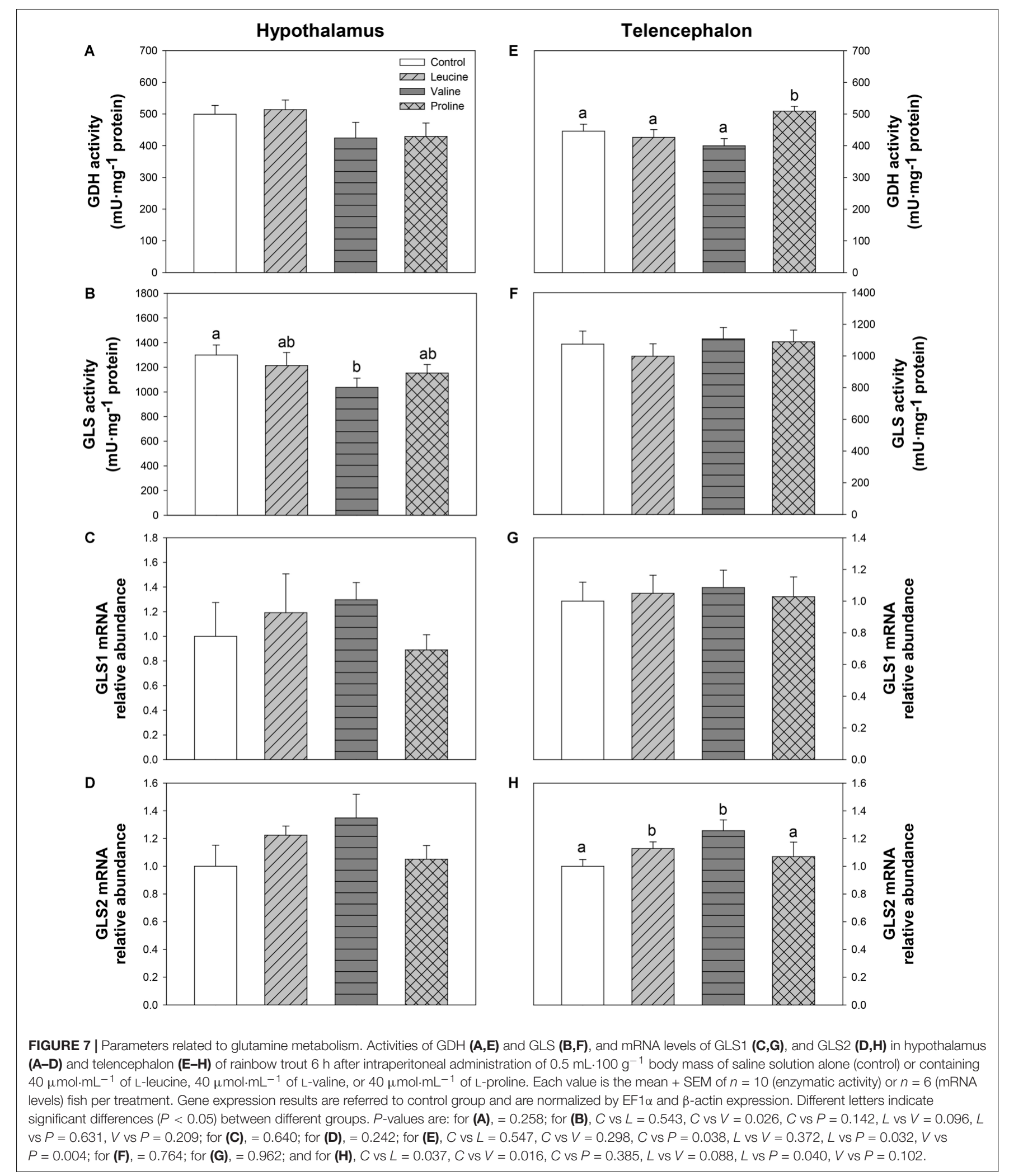

not affect the feeding response, decreased food intake after IP treatment. Thus, at least for the amino acids investigated in the present study, the homeostatic regulation of food intake appears to be independent from the palatability of these nutrients, although further studies, extending this investigation to other amino acids, would be desirable to establish this. 

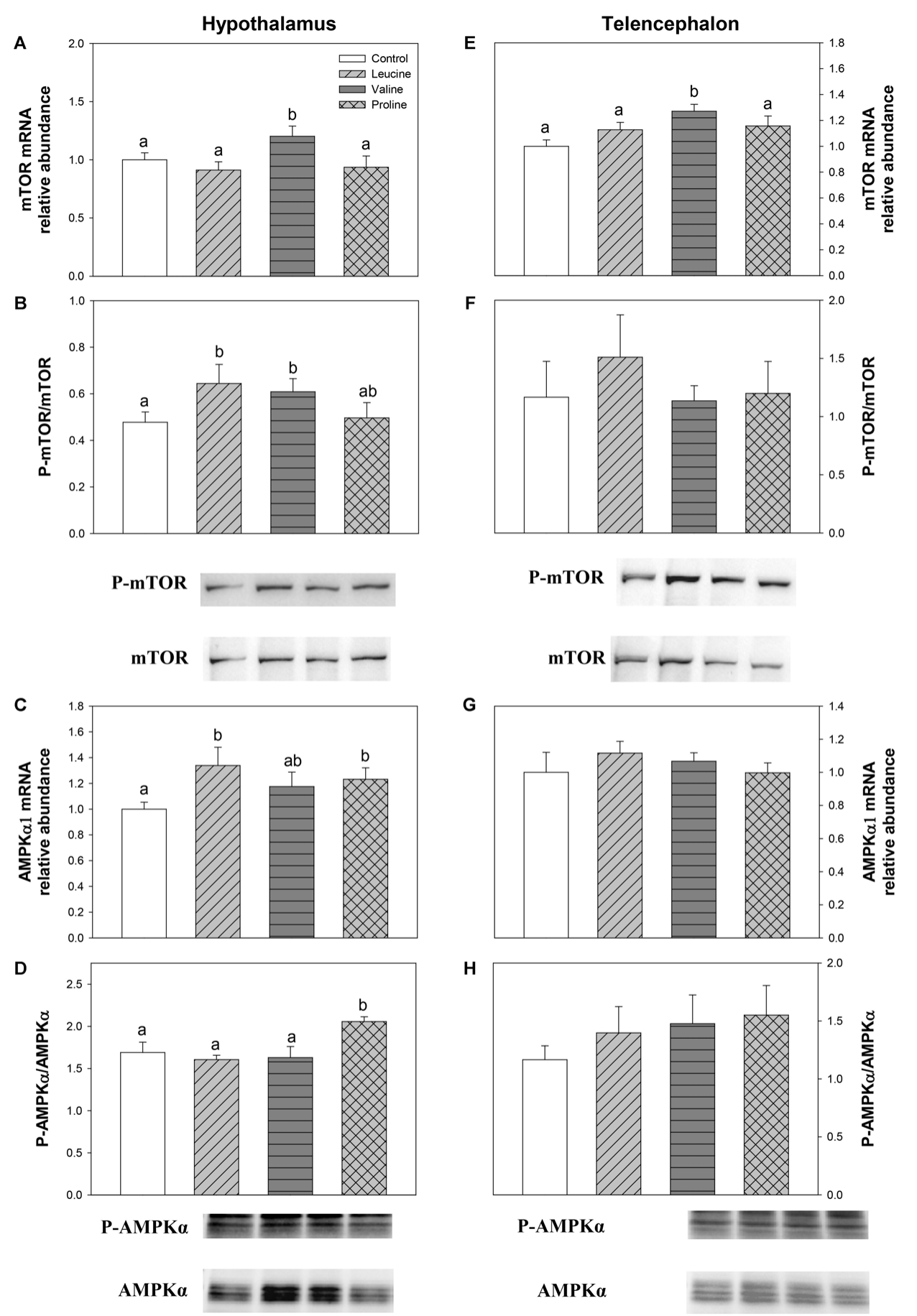

AMPKa $\quad \equiv \equiv \equiv$

FIGURE 8 | Parameters related to mTOR/AMPKa. mRNA levels (A,E) and western blot analysis of phosphorylation status (B,F) of mTOR, and mRNA levels (C,G) and western blot analysis of phosphorylation status (D,H) of AMPKa in hypothalamus (A-D) and telencephalon (E-H) of rainbow trout $6 \mathrm{~h}$ after intraperitoneal administration of $0.5 \mathrm{~mL} \cdot 100 \mathrm{~g}^{-1}$ body mass of saline solution alone (control) or containing $40 \mu \mathrm{mol} \cdot \mathrm{mL}^{-1}$ of L-leucine, $40 \mu \mathrm{mol}^{-} \mathrm{mL}^{-1}$ of L-valine, or $40 \mu \mathrm{mol} \cdot \mathrm{mL}^{-1}$ of L-proline. Each value is the mean + SEM of $n=6$ fish per treatment. Gene expression results are referred to control group and are normalized by EF1 $\alpha$ and $\beta$-actin expression. In western blot analysis, $20 \mu \mathrm{g}$ of total protein were loaded on the gel per lane. Western blots were performed on six individual samples per treatment, and a representative blot per treatment is shown here. Western blot graphs (B,F,D,H) represent the ratio between the phosphorylated protein and the total amount of the target protein. Different letters indicate significant differences $(P<0.05)$ between different groups. $P$-values are: for $(\mathbf{A}), C$ vs $L=0.375, C$ vs $V=0.033, C$ vs $P=0.573, L$ vs $V=0.023, L$ vs $P=0.854, V$ vs $P=0.036$; for (B), $C$ vs $L=0.037, C$ vs $V=0.015, C$ vs $P=0.821, L$ vs $V=0.729$, $L$ vs $P=0.091, V$ vs $P=0.126$; for (C), $C$ vs $L=0.032, C$ vs $V=0.165, C$ vs $P=0.036, L$ vs $V=0.389, L$ vs $P=0.561, V$ vs $P=0.711 ;$ for (D), $C$ vs $L=0.818, C$ vs $V=0.822, C$ vs $P=0.033$, $L$ vs $V=0.537, L$ vs $P<0.001, V$ vs $P=0.032$; for $(\mathbf{E}), C$ vs $L=0.164, C$ vs $V=0.029, C$ vs $P=0.102, L$ vs $V=0.039, L$ vs $P=0.731, V$ vs $P=0.045$; for $(\mathbf{F}),=0.786$; for $(\mathbf{G})=0.681$; and for $(\mathbf{H})=0.638$. 


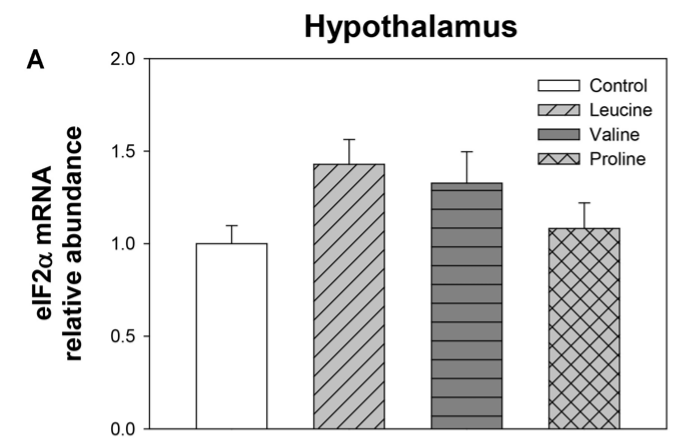

B

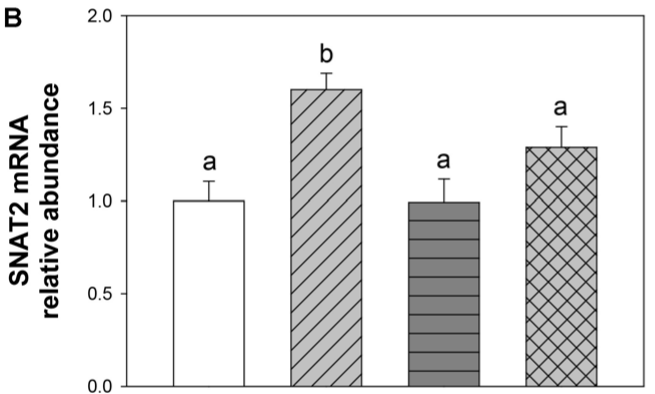

C

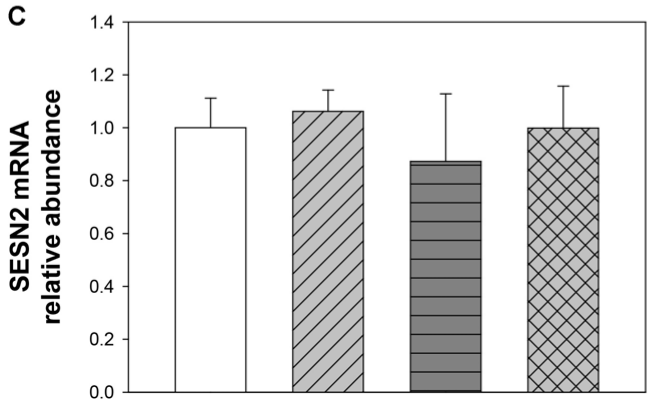

Telencephalon

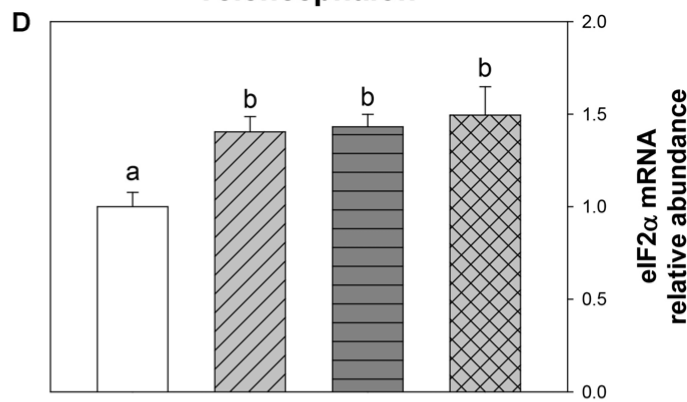

E

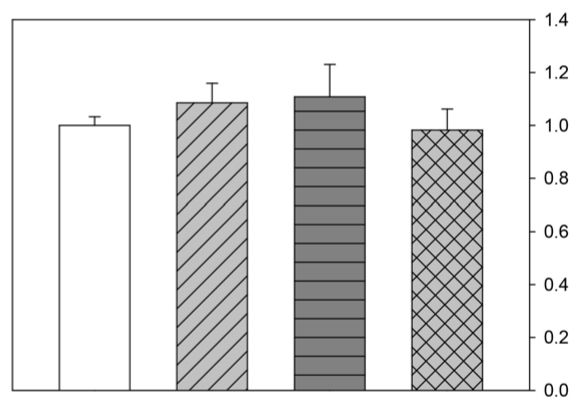

$\mathbf{F}$

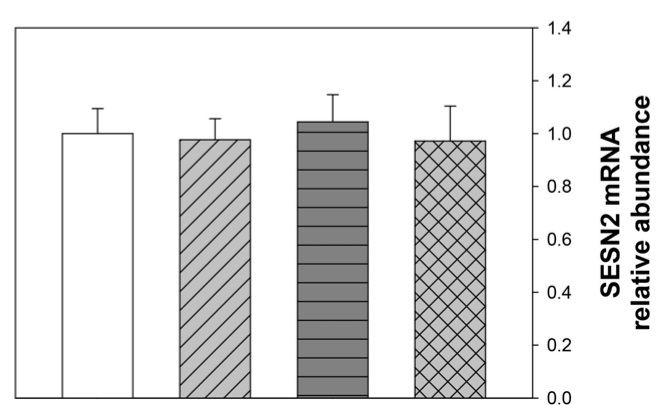

FIGURE 9 | Parameters mediated by GCN2. mRNA levels of elF2 $\alpha$ (A,D), SNAT2 (B,E), and SESN2 (C,F) in hypothalamus (A-C) and telencephalon (D-F) of rainbow trout $6 \mathrm{~h}$ after intraperitoneal administration of $0.5 \mathrm{~mL} \cdot 100 \mathrm{~g}^{-1}$ body mass of saline solution alone (control) or containing $40 \mu \mathrm{mol} \cdot \mathrm{mL}^{-1}$ of L-leucine, $40 \mu \mathrm{mol} \cdot \mathrm{mL}^{-1}$ of L-valine, or $40 \mu \mathrm{mol} \cdot \mathrm{mL}^{-1}$ of L-proline. Each value is the mean $+\mathrm{SEM}$ of $n=6$ fish per treatment. Gene expression results are referred to control group and are normalized by EF1 $\alpha$ and $\beta$-actin expression. Different letters indicate significant differences $(P<0.05)$ between different groups. $P$-values are: for (A), = 0.104; for (B), $C$ vs $L=0.002, C$ vs $V=0.962, C$ vs $P=0.101, L$ vs $V=0.008, L$ vs $P=0.043, V$ vs $P=0.251$; for (C), $=0.882 ;$ for (D), $C$ vs $L=0.014, C$ vs $V=0.025, C$ vs $P=0.018, L$ vs $V=0.850, L$ vs $P=0.803, V$ vs $P=0.662 ;$ for $(\mathbf{E}),=0.691 ;$ and for $(\mathbf{F}),=0.958$

In order to determine the mechanisms underlying the regulation of food intake, we evaluated the expression of neuropeptides (AgRP, NPY, POMC, and CART) involved in the metabolic control of food intake $6 \mathrm{~h}$ after IP treatment with amino acids. In hypothalamus, the anorectic effect of leucine and valine is consistent with the increase in POMC-A1 mRNA abundance produced by both amino acids and the decrease observed in NPY abundance by valine in that area, whereas proline did not induce any significant changes. In general, the response produced by leucine or valine could relate to the homeostatic control of food intake through the expression of neuropeptides in the hypothalamus, in a way similar to that observed after ICV treatment in the same species for leucine but not for valine (Comesaña et al., 2018).

In telencephalon, the increase in mRNA abundance of the orexigenic NPY in trout treated with valine and the downregulation of the anorexigenic POMC-Alafter IP administration of proline is not consistent with changes in food intake. The function of these neuropeptides in fish telencephalon is unknown and, based on this and in our previous ICV study (Comesaña et al., 2018), evidence is starting to accumulate that it is probably different from that in hypothalamus (Soengas et al., 2018), and likely also unrelated to the reward system, at least when amino acids are sensed after IP or ICV administration, by-passing ingestion, and luminal absorption.

\section{Differential Activation of Amino Acid Sensing Systems in Hypothalamus}

Multiple amino acid sensors in different areas of the brain, and particularly in the hypothalamus, can signal changes in concentrations of circulating amino acids and modulate feeding in mammals. In order to determine whether similar mechanisms operate in trout hypothalamus, we evaluated the 


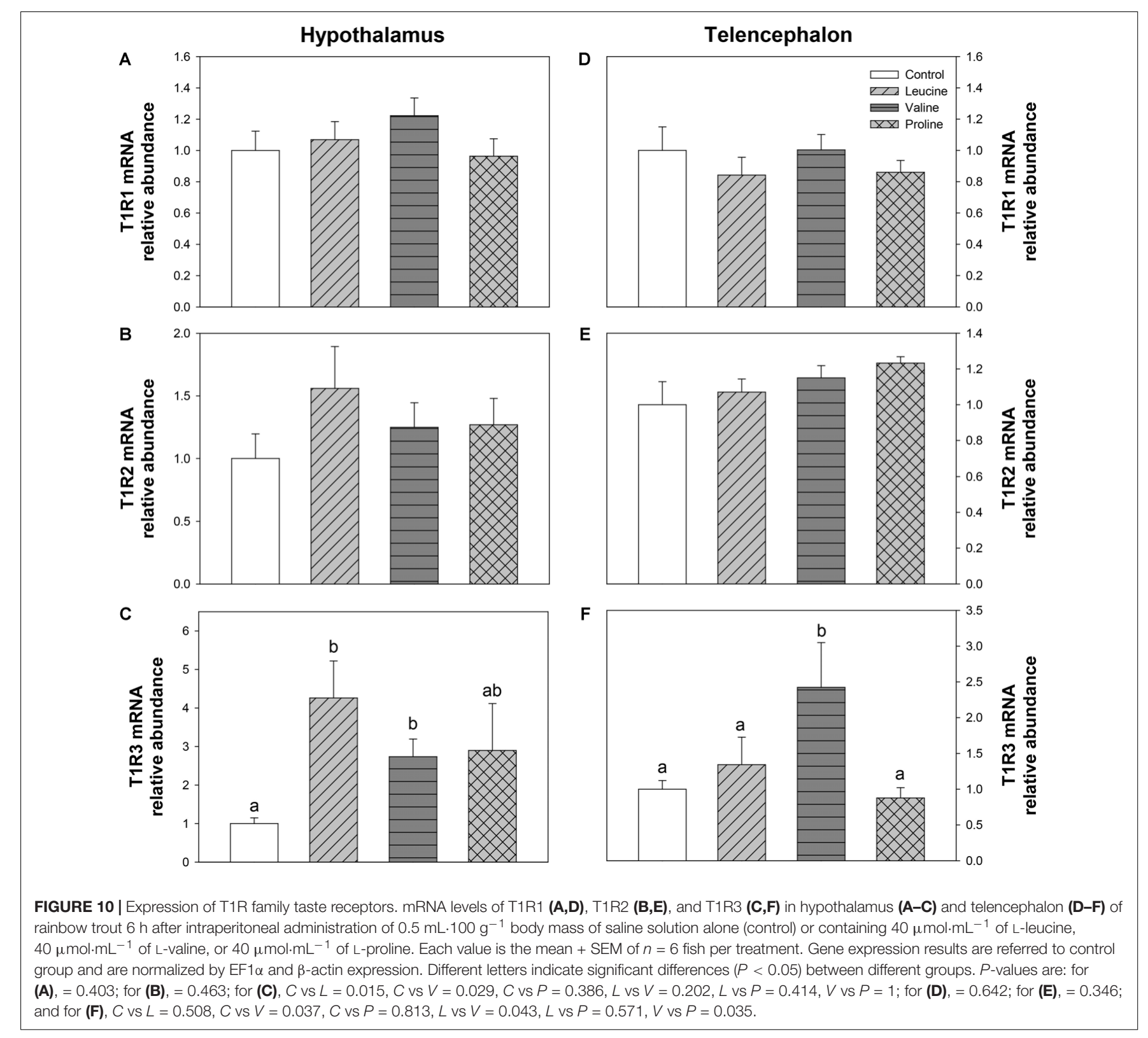

responses of several amino acid sensing systems to a peripheral (post-absorptive) increase in amino acid levels. Furthermore, to establish whether the effects of dietary amino acids are direct, or can be otherwise affected by interactions with other molecules or pathways, hepatic or extra-hepatic metabolism, and/or differential uptake through the blood-brain barrier, we compare results from the present study, after IP administration, with those previously observed after ICV administration of leucine and valine (Comesaña et al., 2018).

The increase in body circulating leucine levels resulted in a higher mRNA abundance of BCKDE2 in this study, which was consistent with previous results after ICV administration (Comesaña et al., 2018), thus supporting the responsiveness of hypothalamic amino acid sensing system dependent on BCAA metabolism. In contrast, IP administration of leucine did not produce any effect on the parameters related to glutamine and glutamate metabolism, while ICV treatment enhanced GLS2 expression (Comesaña et al., 2018). On the other hand, leucine produced an increase in mRNA abundance of T1R3 and a non-significant increase in T1R2, which is in complete agreement with the results we previously observed after ICV treatment (Comesaña et al., 2018). The amino acid sensing system dependent on mTOR also appears to be activated by leucine in rainbow trout hypothalamus since an increased phosphorylation status of this protein was measured after leucine treatment, as observed after ICV treatment (Comesaña et al., 2018). However, we observed an increase in mRNA abundance of AMPK $\alpha 1$ in hypothalamus, which contradicts our previous results where no changes were observed after ICV administration (Comesaña et al., 2018) and mammalian studies, where a rise in leucine levels 

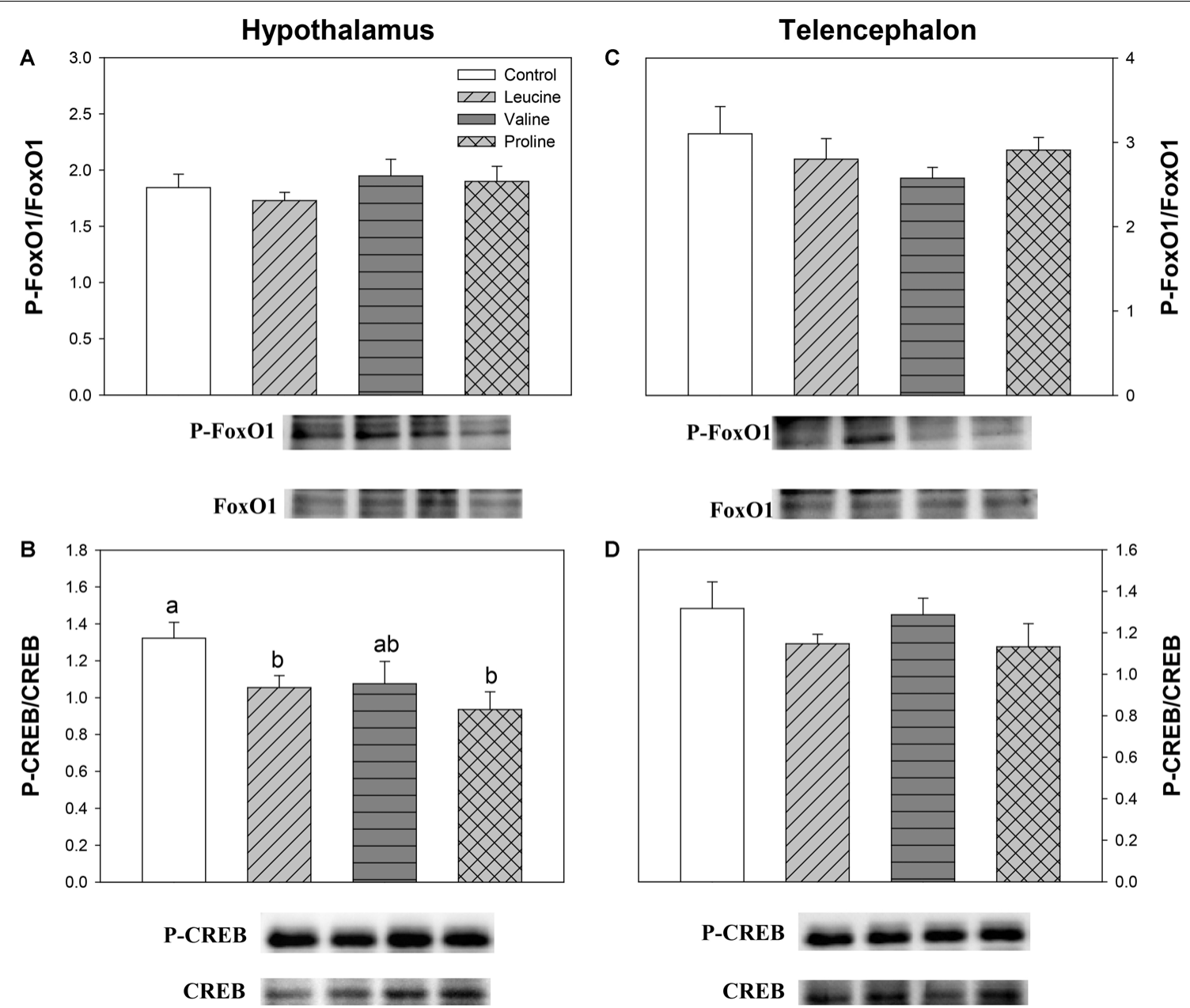

FIGURE 11 | Parameters related to the modulation of transcription factors. Western blot analysis of phosphorylation status of FoxO1 (A,C) and CREB (B,D) in the hypothalamus (A,B) and telencephalon (C,D) of rainbow trout $6 \mathrm{~h}$ after intraperitoneal administration of $0.5 \mathrm{~mL} \cdot 100 \mathrm{~g}^{-1}$ body mass of saline solution alone (control) or containing $40 \mu \mathrm{mol} \cdot \mathrm{mL}^{-1}$ of L-leucine, $40 \mu \mathrm{mol} \cdot \mathrm{mL}^{-1}$ of L-valine, or $40 \mu \mathrm{mol} \cdot \mathrm{mL}^{-1}$ of L-proline. Twenty micrograms of total protein were loaded on the gel per lane. Western blots were performed on six individual samples per treatment, and a representative blot per treatment is shown here. Graphs represent the ratio between the phosphorylated protein and the total amount of the target protein. Each value is the mean + SEM of $n=6$ fish per treatment. Different letters indicate significant differences $(P<0.05)$ between different groups. $P$-values are: for $(\mathbf{A}),=0.655$; for (B), $C$ vs $L=0.038$, C vs $V=0.104, C$ vs $P=0.017, L$ vs $V=0.888, L$ vs $P=0.334, V$ vs $P=0.402 ;$ for $(\mathbf{C}),=0.490$; and for $(\mathbf{D}),=0.341$.

has been reported to induce a decrease in AMPK levels (Cota et al., 2007) and AMPK activity (Ropelle et al., 2008). Since we evaluated the expression of AMPK $\alpha 1$, there is the possibility that AMPK $\alpha 2$ is the form involved in food intake regulation in fish as suggested in mammals (López, 2017). The mechanism dependent on GCN2 also appears to respond to leucine treatment, based on the increase in mRNA abundance of SNAT2, in a way comparable to that observed after ICV treatment (Comesaña et al., 2018). Finally, we have also assessed changes in mRNA abundance of SESN2 for the first time in fish hypothalamus. Although related to the GCN2 kinase pathway, SESN2 can be considered as a leucine sensor. SESN2 negatively regulates mTOR but leucine specifically inactivates SESN2, hence enabling mTOR signaling (Lee et al., 2017; Wolfson and Sabatini, 2017). No changes were observed in SESN2 mRNA abundance after treatment with any of the tested amino acids. In summary, leucine IP treatment generally induced changes in amino acid sensing systems comparable to those we previously observed after ICV administration (Comesaña et al., 2018). In some cases, however, the effects were of lower magnitude and this might relate to the lower levels of leucine in brain after IP, compared to ICV treatment. The fact that results are comparable between IP and ICV treatments allow us to suggest that the action of leucine on sensing mechanisms is direct and might relate to the homeostatic control of food intake in a way comparable to that demonstrated in mammals (Efeyan et al., 2015; Heeley and Blouet, 2016).

Similarly to leucine, valine treatment induced significant effects in mRNA expression and phosphorylation status of mTOR and expression of T1R3 in hypothalamus after IP treatment, but did not affect parameters related to BCAA or glutamine metabolism, and mechanisms related to GCN2. Therefore, even if the effects are not as marked as with leucine, 
valine appears to be able to activate at least some amino acid sensing systems in hypothalamus after IP treatment. These results are inconsistent with those we previously observed after ICV treatment (Comesaña et al., 2018), which suggests that the effects of valine treatment in the present study must result from an indirect action of peripheral valine inducing changes in unknown molecules or mechanisms which in turn signal the hypothalamus.

Discrepancies in the central actions of leucine and valine in fish, in terms of being direct or indirect, could relate to differences in their peripheral metabolism. Despite many similarities, associated with the fact that they are both BCAA, a main distinction which could be of relevance to the results is that leucine is a ketogenic amino acid, while valine is a glucogenic amino acid, as is proline (Jürss and Bastrop, 1995). In fact, differential effects in metabolism have been hinted in this study by the opposite effects observed in glucose and lactate levels in plasma, being glucose significantly reduced in the leucine and proline treatments (compared to the control and valine groups) and lactate reduced in the valine treatment (compared to the control and leucine groups). It is noteworthy that, in mammals, hypothalamic sensing of leucine, valine, and proline is known to modulate glucose metabolism, inhibiting endogenous glucose production (Arrieta-Cruz et al., 2013, 2016). Furthermore, increases in circulating levels of both leucine and proline have also been reported to lower circulating glucose levels (Su et al., 2012; Arrieta-Cruz et al., 2013). Although establishing glucoregulatory effects of central amino acid sensing was not the objective of the present study, it is interesting to note that this mechanism might also be conserved in fish for leucine, while valine again shows a different effect than in mammals.

On the other hand, the possibility for having interactions or antagonisms between different BCAA, particularly when dosed at supra-physiological amounts, has been often reported in mammals, including humans, and birds. In particular, high levels of leucine intake have been described to substantially lower circulating levels of valine and isoleucine but, in contrast, intakes of these BCAA have little influence on the concentration of plasma free leucine (Swendseid et al., 1965; D’Mello and Lewis, 1970). This type of interaction has also been examined in fish, although results are not always consistent (Wilson and Halver, 1986). In a classic study with channel catfish, it was suggested that a nutritional interaction exists between BCAA and that leucine affects the tissue uptake and/or catabolism of valine and isoleucine (Robinson et al., 1984). In rainbow trout, antagonism induced by excess leucine in the diet has also been reported, reducing free valine and isoleucine concentrations in plasma, muscle, and liver, with adverse effects on growth and protein utilization (Yamamoto et al., 2004). More interestingly, excess dietary leucine levels clearly reduced feed intake under self-feeding conditions, which ties well with results from this and our previous study (Comesaña et al., 2018) showing an anorectic effect of leucine administered IP and ICV. However, such interactions cannot explain why valine is also capable of eliciting changes in feed intake in trout, unlike mammals (Heeley and Blouet, 2016), with opposite effects depending on whether it is administered peripherally or centrally. Further studies are clearly needed in order to establish the role of valine in the control of food intake, as well as the mechanism(s) through which it exerts such effects.

The IP administration of proline did not affect most of the hypothalamic amino acid sensors that were assessed, except for an increase in mRNA abundance and phosphorylation status of AMPK $\alpha$, and a decrease in the phosphorylation of CREB. It would therefore seem that either proline or its metabolites are capable of reaching the hypothalamus, as demonstrated also by the significant increase in the concentration of $\alpha$-amino acids in this compartment $6 \mathrm{~h}$ after IP administration, compared to the control, but the effects are likely unrelated to the control of food intake.

\section{Differential Activation of Amino Acid Sensing Systems in Telencephalon}

Treatment with leucine induced changes that in general are compatible with an activation of some amino acid sensing systems in telencephalon. These included parameters related to BCAA metabolism (increased mRNA abundance of BCKDE2), glutamine metabolism (increased mRNA abundance of GLS2) and mediated by GCN2 (increased mRNA abundance of elF2 $\alpha$ ). It seems therefore that leucine is detected in telencephalon in a comparable way to hypothalamus, although more effects were noted when administered ICV (Comesaña et al., 2018). Valine treatment also induced changes that relate to the activation of a few amino acid sensing systems in telencephalon, such as increased mRNA abundance of BCKDE2, BCKDK, GLS2, mTOR, elF2 $\alpha$, and T1R3. The finding that valine elicited more effects than leucine may relate to the higher increase in amino acid levels reached in telencephalon after treatment with valine compared with leucine. As observed in hypothalamus, proline did not exert major effects in telencephalon, despite the increase in total amino acid levels in the area, except an enhancement of GDH enzymatic activity and increased expression of elF2 $\alpha$.

This study confirms that amino acid sensing systems appear to be operative in telencephalon, providing this area with information about circulating levels of amino acids, with different results depending on the type of amino acid. The lack of correlation between the activation of these sensing systems and the feeding stimulation ability of the tested amino acids suggests that these telencephalic mechanisms might relate to still unknown aspects and not to the hedonic control of food intake, as we initially hypothesized (Comesaña et al., 2018).

\section{Possible Mechanisms Linking Amino Acid Sensing and Food Intake Regulation}

In mammals, the activation of nutrient sensing systems influences the production of neuropeptides involved in food intake control through the modulation of transcription factors such as CREB, FoxO1, and BSX (Diéguez et al., 2011; Delgado et al., 2017). However, information on these pathways is still very scarce, especially concerning amino acids.

In the present study, we observed that leucine and proline induced a decrease in the phosphorylation status of CREB in hypothalamus, and a similar trend was noticeable for valine. A similar decrease has been observed in hypothalamus of 
rainbow trout after treatment with other nutrients, like glucose (Otero-Rodiño et al., 2017), oleate or octanoate (Velasco et al., 2017). This transcription factor is known to stimulate NPY/AgRP and to inhibit POMC/CART mRNA abundance (Varela et al., 2011) but changes induced by amino acids might relate to other pathways, considering how all the tested amino acids tended to affect this gene in a comparable manner, including proline, which had no effects on amino acid sensing systems, neuropeptide expression, and food intake after IP administration.

FoxO1 displayed no changes after treatment with any amino acid, in agreement with the lack of effects we also observed after ICV treatment (Comesaña et al., 2018). The phosphorylation status of this transcription factor usually increases after treatment with other nutrients like glucose or fatty acids, as also demonstrated in hypothalamus of rainbow trout (Otero-Rodiño et al., 2017; Velasco et al., 2017). It seems, therefore, that at least part of the mechanisms involved in linking amino acid sensing with the regulation of food intake through changes in the expression of neuropeptides are not the same when comparing amino acids and other nutrients, at least in rainbow trout. In the case of valine, however, the lack of effects on transcription factors which are putatively involved in the control of neuropeptide expression is not surprising, considering that valine did not affect neuropeptide mRNA abundance.

In telencephalon, no changes were observed in transcription factors, supporting our hypothesis that this area is not involved in the homeostatic regulation of food intake. From the coordinated mechanisms responsible for this regulation in hypothalamus (amino acid sensors, transcription factors, and neuropeptide expression), only amino acid sensors appeared to show some response in telencephalon.

\section{Perspectives and Significance}

As a whole, the present and our previous (Comesaña et al., 2018) study provide information about a complex picture of differential effects of amino acids modulating food intake through homeostatic and probably non-homeostatic (presently unknown) mechanisms in central areas (hypothalamus and telencephalon) of rainbow trout. Evidence so far in rainbow trout supports the notion that hypothalamic modulation of food intake through amino acid sensing systems responds to BCAA, and specifically to leucine, in fish as in mammals (Heeley and Blouet, 2016). This could be expected considering that in fish, just as in mammals, the highest correlation between dietary and postprandial systemic free amino acids composition is observed for essential amino acids (Jürss and Bastrop, 1995) and BCAA show the highest changes in plasma amino acid pool in periods of fasting as well as after feeding (Navarro et al., 1997). On the contrary, non-essential amino acids are more quickly metabolized and converted to a great extent. Therefore, BCAA make much more biological sense as indicators of nutrient and energy availability. However, even if amino acid sensing systems are operative in fish and coupled to neuropeptide expression, as in mammals, their regulation of food intake in response to circulating amino acids is clearly different. Contrary to mammals which respond only to leucine, food intake in rainbow trout was also affected by treatment with valine (even if the outcome was different depending on whether it was administered via ICV or IP). Moreover, the action of leucine on sensing mechanisms appears to be direct and relate to the homeostatic control of food intake, while the actions of valine appear to result from an indirect action of peripheral valine inducing changes in unknown molecules or mechanisms which in turn signal the hypothalamus. Further studies are clearly needed in order to establish the role of valine in the control of food intake, as well as the mechanism(s) through which it exerts such effects.

On the other hand, this study confirms that amino acid sensing systems appear to be operative in telencephalon, although the lack of correlation between the activation of these sensing systems and the attractiveness or feeding stimulatory ability of the tested amino acids suggests that these telencephalic mechanisms might relate to still unknown aspects and not to the hedonic control of food intake, as we initially hypothesized (Comesaña et al., 2018). Furthermore, at least for the amino acids investigated in the present study, the homeostatic regulation of food intake appears to be independent on the palatability of these nutrients, at least when amino acids are sensed after IP or ICV administration. We cannot discard, however, that highly palatable amino acids exert hedonic effects by activating the reward system through peripheral (pre-absorptive) actions.

\section{AUTHOR CONTRIBUTIONS}

SC, SM, and JS conceived and designed the research. SC, $\mathrm{CV}$, JM, and MC-S performed the experiments. SC, CV, and MC-S analyzed the data. SC and JS prepared figures. All authors interpreted results of the experiments and drafted, edited, and revised the manuscript, with JS and SM having the main contribution and approving the final version of the manuscript.

\section{FUNDING}

This study was supported by LUCTA and a research grant from Spanish Agencia Estatal de Investigación (AEI) and European Fund for Regional Development (AGL201674857-C3-1-R and FEDER) to JS. SC was a recipient of a predoctoral fellowship from Spanish Ministerio de Educación, Cultura y Deporte (FPU grant reference FPU16/00045). MC-S was a recipient of a postdoctoral fellowship (Program Juan de La Cierva reincorporación) from AEI (IJCI-201630499).

\section{SUPPLEMENTARY MATERIAL}

The Supplementary Material for this article can be found online at: https://www.frontiersin.org/articles/10.3389/fphys. 2018.01209/full\#supplementary-material 


\section{REFERENCES}

Arrieta-Cruz, I., Su, Y., and Gutiérrez-Juárez, R. (2016). Suppression of endogenous glucose production by isoleucine and valine and impact of diet composition. Nutrients 8:79. doi: 10.3390/nu8020079

Arrieta-Cruz, I., Su, Y., Knight, C. M., Lam, T. K. T., and Gutiérrez-Juárez, R. (2013). Evidence for a role of proline and hypothalamic astrocytes in the regulation of glucose metabolism in rats. Diabetes Metab. Res. Rev. 62, 1152-1158. doi: 10.2337/db12-0228

Berthoud, H.-R. (2006). Homeostatic and non-homeostatic pathways involved in the control of food intake and energy balance. Obesity 14(Suppl. 5), 197S-200S. doi: 10.1038/oby.2006.308

Blouet, C., and Schwartz, G. J. (2012). Brainstem nutrient sensing in the nucleus of the solitary tract inhibits feeding. Cell Metab. 16, 579-587. doi: 10.1016/j.cmet. 2012.10.003

Cavanaugh, A. R., Schwartz, G. J., and Blouet, C. (2015). High-fat feeding impairs nutrient sensing and gut brain integration in the caudomedual nucleus of the solitary tract in mice. PLoS One 10:e118888. doi: 10.1371/journal.pone. 0118888

Comesaña, S., Velasco, C., Ceinos, R. M., López-Patiño, M., Míguez, J. M., Morais, S., et al. (2018). Evidence for the presence in rainbow trout brain of amino acid-sensing systems involved in the control of food intake. Am. J. Physiol. Regul. Integr. Comp. Physiol. 314, R201-R215. doi: 10.1152/ajpregu. 00283.2017

Conde-Sieira, M., and Soengas, J. L. (2017). Nutrient sensing systems in fish: impact on food intake regulation and energy homeostasis. Front. Neurosci. 10:603. doi: 10.3389/fnins.2016.00603

Cota, D., Proulx, K., Blake Smith, K. A., Kozma, S. C., Thomas, G., Woods, S. C., et al. (2006). Hypothalamic mTOR signaling regulates food intake. Science 312, 927-930. doi: 10.1126/science.1124147

Cota, D., Proulx, K., and Seeley, R. J. (2007). The role of CNS fuel sensing in energy and glucose production. Gastroenterology 132, 2158-2168. doi: 10.1053/j.gastro. 2007.03.049

de Pedro, N., Pinillos, M. L., Valenciano, A. I., Alonso-Bedate, M., and Delgado, M. J. (1998). Inhibitory effect of serotonin on feeding behavior in goldfish: involvement of CRF. Peptides 19, 505-511. doi: 10.1016/S0196-9781(97) 00469-5

Delgado, M. J., Cerdá-Reverter, J. M., and Soengas, J. L. (2017). Hypothalamic integration of metabolic, endocrine, and circadian signals in fish: involvement in the control of food intake. Front. Neurosci. 11:354. doi: 10.3389/fnins.2017. 00354

Diéguez, C., Vazquez, M. J., Romero, A., López, M., and Nogueiras, R. (2011). Hypothalamic control of lipid metabolism: focus on leptin, ghrelin and melanocortins. Neuroendocrinology 94, 1-11. doi: 10.1159/000328122

D'Mello, J. P. F., and Lewis, D. (1970). Amino acid interactions in chick nutrition. 2. Interrelationships between leucine, isoleucine and valine. Br. Poult. Sci. 11, 313-323. doi: 10.1080/00071667008415821

Efeyan, A., Comb, W. C., and Sabatini, D. M. (2015). Nutrient sensing mechanisms and pathways. Nature 517, 302-310. doi: 10.1038/nature14190

Fromentin, G., Darcel, N., Chaumontet, C., Marsset-Baglieri, A., Nadkarni, N., and Tomé, D. (2012). Peripheral and central mechanisms involved in the control of food intake by dietary amino acids and proteins. Nutr. Res. Rev. 25, 29-39. doi: $10.1017 /$ S0954422411000175

Goh, Y., and Tamura, T. (1980). Olfactory and gustatory responses to amino acids in two marine teleosts - red sea bream and mullet. Comp. Biochem. Physiol. 66C, 217-224. doi: 10.1016/0306-4492(80)90130-6

Hara, T. J. (1994). The diversity of chemical stimulation in fish olfaction and gustation. Rev. Fish Biol. Fish. 4, 1-35. doi: 10.1007/BF00043259

Hara, T. J., Carolsfeld, J., and Kitamura, S. (1999). The variability of the gustatory sensibility in salmonids, with special reference to strain differences in rainbow trout, Oncorhynchus mykiss. Can. J. Fish. Aquat. Sci. 56, 13-24. doi: 10.1139/ f98- 140

Heeley, N., and Blouet, C. (2016). Central amino acid sensing in the control of feeding behavior. Front. Endocrinol. 7:148. doi: 10.3389/fendo.2016.00148

Herrera Moro Chao, D., Argmann, C., Van Eijk, M., Boot, R. G., Ottenhoff, R., Van Roomen, C., et al. (2016). Impact of obesity on taste receptor expression in extra-oral tissues: emphasis on hypothalamus and brainstem. Sci. Rep. 6:29094. doi: $10.1038 /$ srep29094
Hu, F., Xu, Y., and Liu, F. (2016). Hypothalamic roles of mTOR complex I: integration of nutrient and hormone signals to regulate energy homeostasis. Am. J. Physiol. Endocrinol. Metab. 310, E994-E1002. doi: 10.1152/ajpendo. 00121.2016

Hundal, H. S., and Taylor, P. M. (2009). Amino acid transceptors: gate keepers of nutrient exchange and regulators of nutrient signaling. Am. J. Physiol. Endocrinol. Metab. 296, E603-E613. doi: 10.1152/ajpendo.91002.2008

Jewell, J., and Guan, K. (2013). Nutrient signaling to mTOR and cell growth. Trends Biochem. Sci. 38, 233-242. doi: 10.1016/j.tibs.2013.01.004

Jones, K. (1989). The palatability of amino acids and related compounds to rainbow trout, Salmo gairdneri Richardson. J. Fish Biol. 34, 149-160. doi: 10.1111/j.10958649.1989.tb02964.x

Jordi, J., Herzog, B., Camargo, S. M. R., Boyle, C. N., Lutz, T. A., and Verrey, F. (2013). Specific amino acids inhibit food intake via the area postrema or vagal afferents. J. Physiol. 591, 5611-5621. doi: 10.1113/jphysiol.2013.258947

Jürss, K., and Bastrop, K. (1995). “Amino acid metabolism in fish," in Biochemistry and Molecular Biology of Fishes, Vol. 4, eds P. W. Hochachka and T. P. Mommsen (San Diego, CA: Elsevier Science B.V), 159-189.

Kamalam, B. S., Medale, F., Kaushik, S., Polakof, S., Skiba-Cassy, S., and Panserat, S. (2012). Regulation of metabolism by dietary carbohydrate in two lines of rainbow trout divergently selected for muscle fat content. J. Exp. Biol. 215, 2567-2578. doi: 10.1242/jeb.070581

Kohbara, J., and Caprio, J. (2001). Taste responses of the facial and glossopharyngeal nerves to amino acids in the rainbow trout. J. Fish Biol. 58, 1062-1072. doi: 10.1111/j.1095-8649.2001.tb00555.x

Lee, A. A., and Owyang, C. (2017). Sugars, sweet taste receptors, and brain responses. Nutrients 9, 653. doi: 10.3390/nu9070653

Lee, J. H., Cho, U. S., and Karin, M. (2017). Sestrin regulation of TORC1: is sestrin a leucine sensor? Sci. Signal. 9:re5. doi: 10.1126/scisignal.aaf2885

Li, P., Mai, K., Trushenski, J., and Wu, G. (2009). New developments in fish amino acid nutrition: towards functional and environmentally oriented aquafeeds. Amino Acids 37, 43-53. doi: 10.1007/s00726-008-0171-1

Librán-Pérez, M., Geurden, I., Dias, K., Corraze, G., Panserat, S., and Soengas, J. L. (2015). Feeding rainbow trout with a lipid-enriched diet: effects on fatty acid sensing, regulation of food intake and cellular signaling pathways. J. Exp. Biol. 218, 2610-2619. doi: 10.1242/jeb.123802

López, M. (2017). Hypothalamic AMPK: a golden target against obesity? Eur. J. Endocrinol. 176, R235-R246. doi: 10.1530/EJE-16-0927

Lutter, M., and Nestler, E. J. (2009). Homeostatic and hedonic signals interact in the regulation of food Intake. J. Nutr. 139, 629-632. doi: 10.3945/jn.108.097618

Marui, T., Evans, R. E., Zielinski, B., and Hara, T. J. (1983). Gustatory responses of the rainbow trout (Salmo gairdneri) palate to amino acids and derivatives. J. Comp. Physiol. 153, 423-433. doi: 10.1007/BF00612597

Moore, S. (1968). Amino acid analysis: aqueous dimethyl sulfoxide as solvent for the ninhydrin reaction. J. Biol. Chem. 243, 6281-6283.

Morais, S. (2017). The physiology of taste in fish: potential implications for feeding stimulation and gut chemical sensing. Rev. Fish. Sci. Aquac. 25, 133-149. doi: 10.1080/23308249.2016.1249279

Morrison, C. D., and Laeger, T. (2015). Protein-dependent regulation of feeding and metabolism. Trends Endocrinol. Metab. 26, 256-262. doi: 10.1016/j.tem. 2015.02.008

Mueller, T., and Wullimann, M. F. (2009). An evolutionary interpretation of teleostean forebrain anatomy. Brain Behav. Evol. 74, 30-42. doi: 10.1159/ 000229011

Navarro, I., Blasco, J., Baños, N., and Gutiérrez, J. (1997). Effects of fasting and feeding on plasma amino acid levels in brown trout. Fish Physiol. Biochem. 16, 303-309. doi: 10.1023/A:1007759316656

O'Connell, L., and Hofmann, H. (2011). The vertebrate mesolimbic reward system and social behavior network: a comparative synthesis. J. Comp. Neurol. 519, 3599-3639. doi: 10.1002/cne.22735

Oike, H., Nagai, T., Furuyama, A., Okada, S., Aihara, Y., Ishimaru, Y., et al. (2007). Characterization of ligands for fish taste receptors. J. Neurosci. 27, 5584-5592. doi: 10.1523/JNEUROSCI.0651-07.2007

Otero-Rodiño, C., Velasco, C., Álvarez-Otero, R., López Patiño, M. A., Míguez, J. M., and Soengas, J. L. (2017). Changes in the levels and phosphoprylation status of Akt, AMPK, CREB and FoxO1 in hypothalamus of rainbow trout under conditions of enhanced glucosensing activity. J. Exp. Biol. 220, 4410-4417. doi: 10.1242/jeb.165159 
Papatryphon, E., and Soares, J. (2000). Identification of feeding stimulants for striped bass, Morone saxatilis. Aquaculture 185, 339-352. doi: 10.1016/S00448486(99)00347-6

Pfaffl, M. W. (2001). A new mathematical model for relative quantification in real-time RT-PCR. Nucleic Acids Res. 29:e45. doi: 10.1093/nar/29.9.e45

Polakof, S., Míguez, J. M., and Soengas, J. L. (2008a). Dietary carbohydrates induce changes in glucosensing capacity and food intake in rainbow trout. Am. J. Physiol. Reg. Integr. Comp. Physiol. 295, R478-R489. doi: 10.1152/ajpregu. 00176.2008

Polakof, S., Panserat, S., Plagnes-Juan, E., and Soengas, J. L. (2008b). Altered dietary carbohydrates significantly affect gene expression of the major glucosensing components in Brockmannn bodies and hypothalamus of rainbow trout. Am. J. Physiol. Reg. Integr. Comp. Physiol. 295, R1077-R1088. doi: 10.1152/ajpregu. 90476.2008

Robinson, E. H., Poe, W. E., and Wilson, R. P. (1984). Effects of feeding diets containing an imbalance of branched-chain amino acids on fingerling channel catfish. Aquaculture 37, 51-62. doi: 10.1016/0044-8486(84)90043-7

Ropelle, E., Pauli, J., Fernandes, M., Rocco, S., Marin, R., Morari, J., et al. (2008). Central role for neuronal AMP-activated protein kinase (AMPK) and mammalian target of rapamycin (mTOR) in high-protein diet-induced weight loss. Diabetes Metab. Res. Rev. 57, 594-605. doi: 10.2337/db07-0573

Rossi, M. A., and Stuber, G. D. (2017). Overlapping brain circuits for homeostatic and hedonic feeding. Cell Metab. 27, 42-56. doi: 10.1016/j.cmet.2017.09.021

Sánchez-Gurmaches, J., Cruz-Garcia, L., Gutiérrez, J., and Navarro, I. (2010). Endocrine control of oleic acid and glucose metabolism in rainbow trout (Oncorhynchus mykiss) muscle cells in culture. Am. J. Physiol. Reg. Integr. Comp. Physiol. 299, R562-R572. doi: 10.1152/ajpregu.00696.2009

Skiba-Cassy, S., Lansard, M., Panserat, S., and Médale, F. (2009). Rainbow trout genetically selected for greater muscle fat content display increased activation of liver TOR signaling and lipogenic gene expression. Am. J. Physiol. Reg. Integr. Comp. Physiol. 297, R1421-R1429. doi: 10.1152/ajpregu.00312.2009

Soengas, J. L., Cerdá-Reverter, J. M., and Delgado, M. J. (2018). Central regulation of food intake in fish: an evolutionary perspective. J. Mol. Endocrinol. 60, R171-R199. doi: 10.1530/JME-17-0320

Su, Y., Lam, T. K. T., He, W., Pocai, A., Bryan, J., Aguilar-Bryan, L., et al. (2012). Hypothalamic leucine metabolism regulates liver glucose production. Diabetes Metab. Res. Rev. 61, 85-93. doi: 10.2337/db11-0857

Swendseid, M. E., Villalobos, J., Figueroa, W. S., and Drenick, E. J. (1965). The effects of test doses of leucine, isoleucine or valine on plasma amino acid levels. The unique effect of leucine. Am. J. Clin. Nutr. 17, 317-321. doi: 10.1093/ajcn/ 17.5.317

Varela, L., Vázquez, M. J., Cordido, F., Nogueiras, R., Vidal-Puig, A., Diéguez, C., et al. (2011). Ghrelin and lipid metabolism: key partners in energy balance. J. Mol. Endocrinol. 46, R43-R63. doi: 10.1677/JME-100068

Velasco, C., Librán-Pérez, M., Otero-Rodiño, C., López-Patiño, M. A., Míguez, J. M., and Soengas, J. L. (2016). Ceramides are involved in the regulation of food intake in rainbow trout (Oncorhynchus mykiss). Am. J. Physiol. Reg. Integr. Comp. Physiol. 311, R658-R668. doi: 10.1152/ajpregu.00201.2016

Velasco, C., Otero-Rodiño, C., Comesaña, S., Míguez, J. M., and Soengas, J. L. (2017). Hypothalamic mechanisms linking fatty acid sensing and food intake regulation in rainbow trout. J. Mol. Endocrinol. 59, 377-390. doi: 10.1530/JME17-0148

Wacyk, J., Powell, M., Rodnick, K., Overturf, K., Hill, R., and Hardy, R. (2012). Dietary protein source significantly alters growth performance, plasma variables and hepatic gene expression in rainbow trout (Oncorhynchus mykiss) fed amino acid balanced diets. Aquaculture 356-357, 223-234. doi: 10.1016/j.aquaculture. 2012.05.013

Wauson, E., Zaganjor, E., Lee, A., Guerra, M., Ghosh, A., Bookout, A., et al. (2012). The G protein-coupled taste receptor T1R1/T1R3 regulates mTORC1 and autophagy. Mol. Cell 47, 851-862. doi: 10.1016/j.molcel.2012.08.001

Wilson, R. P., and Halver, J. E. (1986). Protein and amino acids requirements of fishes. Annu. Rev. Nutr. 6, 225-244. doi: 10.1146/annurev.nu.06.070186.001301

Wolfson, R. L., and Sabatini, D. M. (2017). The dawn of the age of amino acid sensors for the mTORC1 pathway. Cell Metab. 26, 301-309. doi: 10.1016/j.cmet. 2017.07.001

Wu, G., Bazer, F. W., Burghardt, R. C., Johnson, G. A., Kim, S. W., Knabe, D. A., et al. (2011). Proline and hydroxyproline metabolism: implications for animal and human nutrition. Amino Acids 40, 1053-1063. doi: 10.1007/s00726-0100715-z

Yamamoto, T., Shima, T., and Furuita, H. (2004). Antagonistic effects of branchedchain amino acids induced by excess protein-bound leucine in diets for rainbow trout (Oncorhynchus mykiss). Aquaculture 232, 539-550. doi: 10.1016/S00448486(03)00543-X

Conflict of Interest Statement: The authors declare that the research was conducted in the absence of any commercial or financial relationships that could be construed as a potential conflict of interest.

Copyright (C) 2018 Comesaña, Velasco, Conde-Sieira, Míguez, Soengas and Morais. This is an open-access article distributed under the terms of the Creative Commons Attribution License (CC BY). The use, distribution or reproduction in other forums is permitted, provided the original author(s) and the copyright owner(s) are credited and that the original publication in this journal is cited, in accordance with accepted academic practice. No use, distribution or reproduction is permitted which does not comply with these terms. 